\title{
Optical, Photoluminescent, and Photoconductive Properties of Novel High- Performance Organic Semiconductors
}

\author{
Oksana Ostroverkhova, Andrew D. Platt and Whitney E. B. Shepherd \\ Oregon State University
}

USA

\section{Introduction}

Organic semiconductors have been investigated as an alternative to inorganic semiconductors due to their low cost, ease of fabrication, and tunable properties (Forrest, 2004). Applications envisioned for organic semiconductors include xerography, thin-film transistors, light-emitting diodes, solar cells, organic lasers, and many others (Peumans et al., 2003; Murphy \& Frechet, 2007; Samuel \& Turnbull, 2007). Since most of these applications rely on the conductive and photoconductive properties of the materials, it is important to understand physical mechanisms of charge photogeneration, transport, trapping, and recombination. However, despite numerous theoretical and experimental studies of the optical and electronic properties of organic solids, these mechanisms are not well understood and are still the subject of debate in the literature (Sariciftci, 1997; Podzorov et al., 2004; Moses et al., 2006; Troisi \& Orlandi, 2006; Coropceanu et al., 2007; Cheng \& Silbey, 2008; Laarhoven et al., 2008). Indeed, it is a complicated task to reveal and utilize the intrinsic properties of organic materials, since they are often masked by the influence of impurities, the presence of which is sensitive to the methods of material purification and device fabrication. As a result, measurements performed in the same material using different experimental techniques often provide conflicting results (Nelson et al., 1998; Hegmann et al., 2002; Podzorov et al., 2003; Jurchescu et al., 2004; Lang et al., 2004; Thorsmølle et al., 2004; Ostroverkhova et al., 2005a; Ostroverkhova et al., 2006a; Koeberg et al., 2007; Laarhoven et al., 2008; Najafov et al., 2008; Marciniak et al., 2009). Experimental methods that probe charge carrier dynamics on picosecond (ps) time-scales after a 100femtosecond ( $\mathrm{fs}$ ) pulsed photoexcitation have had most success in revealing intrinsic properties of organic semiconductors (Hegmann et al., 2002; Thorsmølle et al., 2004; Hegmann et al., 2005; Ostroverkhova et al., 2005a; Ostroverkhova et al., 2005b). In contrast, techniques that probe equilibrium charge transport are much more sensitive to extrinsic effects (Nelson et al., 1998; Knipp et al., 2003; de Boer et al., 2004; Jurchescu et al., 2004). However, since properties under equilibrium conditions are relevant for most devices, it is necessary to understand how they are related to intrinsic properties and find ways to improve materials and device fabrication techniques in order to minimize extrinsic effects. Therefore, one of the emphases of this chapter is on photoexcited charge carrier dynamics from sub-ps (non-equilibrium) to many seconds (equilibrium) after photoexcitation in a variety of organic crystals and thin films (Sections 3.3-3.4).

Source: Advances in Lasers and Electro Optics, Book edited by: Nelson Costa and Adolfo Cartaxo, ISBN 978-953-307-088-9, pp. 838, April 2010, INTECH, Croatia, downloaded from SCIYO.COM 
Of particular technological interest are small-molecular-weight solution-processable materials that can be cast into high-performance (photo)conductive thin films. Functionalized anthradithiophene (ADT) and pentacene derivatives have attracted considerable attention due to their high charge carrier mobility, photoconductivity, and luminescence. In particular, charge carrier (hole) mobilities of over $1.2 \mathrm{~cm}^{2} / \mathrm{Vs}$ have been observed in thin-film transistors (TFTs) based on solution-deposited films of these materials (Park et al., 2007; Park et al., 2008). Slight chemical modifications of the side groups of both ADT and pentacene derivatives lead to considerable changes in molecular packing, which affect electronic and optical properties of thin films (Platt et al., 2009a; Platt et al., 2009b; Platt et al., 2009c). Additional changes in these properties may be produced by functionalization of the core of the molecule. In Sections 3.1-3.5, we will summarize optical, photoluminescent (PL), and (photo)conductive properties of several functionalized ADT and pentacene derivatives. In addition to a promising potential of ADT pristine compounds for (opto)electronic applications, the availability of these high-performance, solutionprocessable, structurally similar, derivatives with different highest occupied molecular orbital (HOMO) and lowest unoccupied molecular orbital (LUMO) energies can be utilized to create composites with optical and electronic properties tailored for specific applications. In Sections 3.1.3 and 3.3.2, we will describe how charge carrier dynamics in ADT films can be manipulated using competition between charge and energy transfer achieved by introducing various guest molecules into an ADT host (Day et al., 2009a). An ultimate understanding of the charge carrier and exciton dynamics in organic semiconductors can be reached, however, only if relationships between charge transfer and energy transfer rates on the molecular level and macroscopic (photo) conductivity in the bulk material are established. Our preliminary studies of properties of individual molecules are presented in Section 3.2.

Finally, most devices that utilize (opto) electronic properties of materials require application of static electric fields with deposited electrodes. The processes occurring at the metalorganic interfaces at the electrodes can significantly affect the performance of a device (Brutting, 2005; Day et al., 2009b), introducing another difficulty in the materials characterization and another variable into the device performance. Effects of the electrode material on the (photo)conductive performance of organic thin-film devices, at various timescales after photoexcitation, will be briefly reviewed in Section 3.6.

\section{Experimental}

\subsection{Materials and sample preparation}

In our studies of non-equilibrium charge carrier dynamics at sub-ps time scales after 100-fs photoexcitation (Section 3.3.1), we used single crystals of ultra-high-purity pentacene (Pc) (Jurchescu et al., 2004), pentacene derivative functionalized with 6,13-bis (triisopropylsilylethynyl) (TIPS) side groups (TIPS-pentacene) (Hegmann et al., 2002; Ostroverkhova et al., 2005a; Ostroverkhova et al., 2005b; Anthony, 2006; Ostroverkhova et al., 2006a), rubrene (Rub) (Podzorov et al., 2003; Podzorov et al., 2004), and tetracene (Tc) (Moses et al., 2006) (Fig.1). The Pc powder was purified using vacuum sublimation under a temperature gradient (Jurchescu et al., 2004). Rub, Tc and Pc single crystals were obtained using physical vapor transport techniques as described in Jurchescu et al., 2004 and Podzorov et al., 2003. The TIPS-pentacene single crystals were grown in a saturated tetrahydrofuran (THF) solution at $4^{\circ} \mathrm{C}$ (Anthony et al., 2001). Eight TIPS-pentacene crystals 
and four Pc, Rub and Tc crystals (each) were used in these experiments and yielded similar results (Ostroverkhova et al., 2006a). For the same experiments, we also prepared polycrystalline Pc and TIPS-pentacene thin films. All of the Pc and some TIPS-pentacene films were prepared on mica, glass, or $\mathrm{KCl}$ substrates by thermal evaporation of the corresponding powder heated to $200-250^{\circ} \mathrm{C}$ in high vacuum $\left(10^{-6}-10^{-7} \mathrm{Torr}\right)$ at a deposition rate of $0.3 \AA /$ s. The thickness of the films was measured with a profilometer (Tencor Instruments) and ranged between $150 \mathrm{~nm}$ and $1.3 \mu \mathrm{m}$ (Ostroverkhova et al., 2005a; Ostroverkhova et al., 2005b). TIPS-pentacene films drop-cast from THF solution at room temperature were also prepared.

In our studies of anisotropy of the transient photoconductivity (Section 3.3.1), we used single crystals of pentacene functionalized with: (i) TIPS and (ii) 6,13-bis (triethylsilylethynyl) (TES) side groups. The single crystals had dimensions typically around (1.5-2) $\mathrm{mm} \times(2-4) \mathrm{mm}$ (as illustrated in Fig.9(c)) with a thickness of 300-500 $\mu \mathrm{m}$. Although both TIPS-pentacene and TES-pentacene crystals are triclinic (with the unit cell parameters listed in Table 1), the molecular packing and resulting $\pi$-overlap in these crystals is different, as the TIPS-pentacene crystals assume a more two-dimensional (2D) "brick-wall"-type structure, while the TES-pentacene crystals exhibit a one-dimensional (1D) "slipped-stack"type structure (Figs.9(a) and (b), respectively)(Anthony, 2006). Our crystallographic analysis showed that the largest area crystal surface corresponds to the $a-b$ plane of the crystals, with the $a$-axis parallel $(||)$ to the long axis in both TIPS-pentacene and TES-pentacene crystals. TIPS-pentacene and TES-pentacene crystals have almost identical absorption spectra in solution, with the absorption edge at around $700 \mathrm{~nm}$, which shifts to $\sim 850 \mathrm{~nm}$ in a crystal. Eight TIPS and four TES crystals were used in these experiments (Ostroverkhova et al., 2006b).

\begin{tabular}{|c|c|c|c|c|c|c|}
\hline Compound & $\mathrm{a}(\AA)$ & $\mathrm{b}(\AA)$ & $\mathrm{c}(\AA)$ & $\alpha(\mathrm{deg})$ & $\beta(\mathrm{deg})$ & $\gamma(\mathrm{deg})$ \\
\hline TIPS-pent. & 7.565 & 7.75 & 16.835 & 89.15 & 78.42 & 83.63 \\
\hline TES-pent. & 7.204 & 9.994 & 11.326 & 80.81 & 89.13 & 82.21 \\
\hline ADT-TES-F & 7.71 & 7.32 & 16.35 & 87.72 & 89.99 & 71.94 \\
\hline ADT-TIPS-F & 7.58 & 8.18 & 16.15 & 100.85 & 92.62 & 98.79 \\
\hline
\end{tabular}

Table 1. Unit cell parameters for functionalized pentacene and ADT derivatives used in our studies.

In our studies of photophysical and photoconductive properties on time-scales from subnanoseconds (ns) after 100 fs excitation to an equilibrium (Sections 3.1, 3.3.2, 3.4, and 3.5), we explored thin films of TIPS-pentacene, of fluorinated ADT derivatives functionalized with TES and TIPS side groups (ADT-TES-F and ADT-TIPS-F, respectively), and of another ADT derivative functionalized with TIPS side-groups, ADT-TIPS-CN (Figure 1) (Day et al., 2008; Day et al., 2009a; Day et al., 2009b; Platt et al., 2009b). In addition to pristine materials, we explored films of ADT-TES-F doped with various concentrations of $\mathrm{C}_{60}$, TIPS-pentacene, or ADT-TIPS-CN. Stock solutions of functionalized ADT derivatives were prepared at $\sim 1 \%$ by weight in toluene. For solution measurements, solutions were prepared by dilution of stock solutions to $\sim 10^{-4} \mathrm{M}$. Films with thickness of $1-2 \mu \mathrm{m}$ were prepared by drop-casting stock solutions onto glass substrates at $\sim 60{ }^{\circ} \mathrm{C}$. Composite films were similarly prepared 
from stock solutions of known mixtures of ADT-TES-F and $\mathrm{C}_{60}$, TIPS-pentacene, or ADTTIPS-CN. When dealing with pristine compounds, this preparation method yielded polycrystalline ADT-TES-F, ADT-TIPS-F, and TIPS-pentacene and amorphous ADT-TIPS$\mathrm{CN}$ films (as confirmed by X-ray diffraction and transmission electron microscopy (TEM)). The unit cell parameters for the fluorinated ADT derivatives studied are listed in Table 1. For measurements of dark current and photoresponse, glass substrates were prepared by photolithographic deposition of either $5 \mathrm{~nm} / 50 \mathrm{~nm}$-thick $\mathrm{Cr} / \mathrm{Au}$ or $100 \mathrm{~nm}$-thick aluminum (Al) electrode pairs. Each pair consisted of 10 interdigitated finger pairs, with $1 \mathrm{~mm}$ finger length, $25 \mu \mathrm{m}$ finger width and $25 \mu \mathrm{m}$ gaps between the fingers of opposite electrodes. Films were drop-cast onto the interdigitated regions. Films on coplanar electrodes with 25 or 50 $\mu \mathrm{m}$ gap were also prepared.

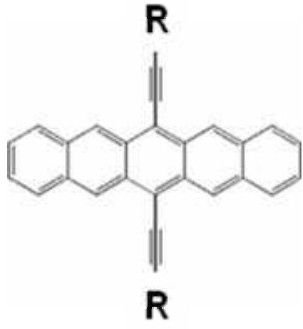

(a)

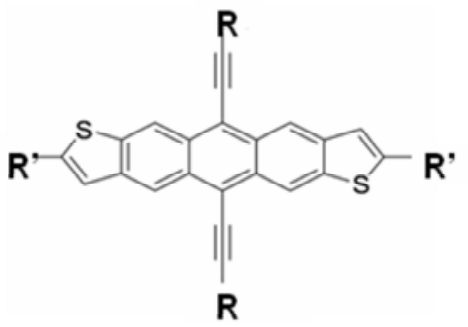

(b)

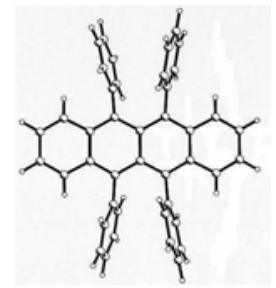

(c)

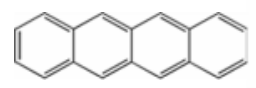

(d)

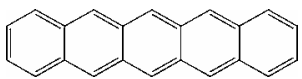

(e)

Fig. 1. Molecular structures of (a) functionalized pentacene, (b) functionalized ADT, (c) rubrene (Rub), (d) tetracene (Tc), and (e) pentacene (Pc). R = TIPS or TES, $\mathrm{R}^{\prime}=\mathrm{F}$ or CN.

\subsection{Measurements of optical and photophysical properties}

Optical absorption spectra were measured using a halogen lamp and a fiber-coupled Ocean Optics USB2000 spectrometer. Absorbance A was calculated from the incident $\left(\mathrm{I}_{0}\right)$ and transmitted (I) beam intensities as $A=-\log \left(I / I_{0}\right)$. Reflection losses were taken into account by referencing with respect to cuvettes with pure solvent or clean glass substrates for solution and film measurements, respectively. Emission spectra were acquired in a custom fluorescence measurement setup with laser excitation at wavelengths of either $400 \mathrm{~nm}$ (frequency-doubled mode-locked Ti:Sapphire laser from KM Labs) or $532 \mathrm{~nm}$ (Nd:YVO laser from Coherent, Inc.). Emitted photons were collected using a parabolic mirror and detected with a fiber coupled spectrometer (Ocean Optics USB2000 calibrated against a 3100 $\mathrm{K}$ black-body emitter). Absorption of solutions was measured using a standard $1 \mathrm{~cm}$ path length quartz cuvette with a halogen light source fiber-optically delivered to the sample holder and spectrometer. Photoluminescence (PL) quantum yields (QYs) in solution were referenced against standards with known quantum yields and corrected for differences in optical density and solvent refractive index. The ADT derivatives were measured against rhodamine 6G in ethanol $(\Phi=0.95)$ and DCDHF-N-6 in toluene $(\Phi=0.85)$ (Lord et al., 2007). The QY of TIPS pentacene solution was measured against rhodamine 6G in ethanol and 
Alexa Fluor 647 in a phosphate buffer solution ( $\mathrm{pH} 7.2, \Phi=0.33$ ). The QYs in films were estimated using DCDHF-N-6 in polymethylmethacrylate (PMMA) $(\Phi=0.98)$ as a reference and assuming a value of 1.7 for the index of refraction (Lord et al., 2007). The detection limit of the setup was estimated to be at $\Phi \approx 0.5 \%$ at $650 \mathrm{~nm}$.

PL lifetime measurements were performed using a mode-locked Ti:Sapphire laser frequency-doubled with a beta-barium borate (BBO) crystal with a repetition rate of $93 \mathrm{MHz}$ picked at $9.3 \mathrm{MHz}$ using a home-built pulse picker (based on a $\mathrm{TeO}_{2}$ acousto-optic modulator from NEOS) and 80-fs pulses as the excitation source. A single-photon avalanche photodiode (SPAD - Molecular Photonic Devices) was used in conjunction with a timecorrelated single-photon counter (TCSPC) data analysis board (PicoQuant TimeHarp 200) for detection. The instrument response function (IRF) ( 200 ps) was recorded using scattered light from an etched microscope slide. For measurements of the temperature dependence of film spectra, samples on pre-cut microscope slides were mounted on a custom built electrically heated and water cooled stage (range: $278-360 \mathrm{~K}$ ) for temperature control. PL measurements were taken in situ over the entire temperature range, in ambient air. Similar experiments were previously performed under $\mathrm{N}_{2}$ atmosphere and showed no discernable difference.

For measurements of electric field-induced PL quenching, either $532 \mathrm{~nm} \mathrm{cw}$ light or pulsed $400 \mathrm{~nm}$ light was focused on the samples similar to those used in our (photo)conductivity studies. At $532 \mathrm{~nm} \mathrm{cw}$ photoexcitation, PL was collected using a Thorlabs amplified photodetector and a SRS830 lock-in amplifier. Keithley 237 source-measure unit was used to apply voltage in the range of $0-500 \mathrm{~V}$ and measure photocurrent, simultaneously with measurements of the PL. At $400 \mathrm{~nm}$ pulsed excitation, PL transient decay was detected using SPAD and TCSPC, as described above. The experiment was repeated at voltages up to $500 \mathrm{~V}$ and at different temperatures.

\subsection{Single-molecule-level PL imaging}

Samples for PL imaging at the single-molecule level (Section 3.2) were prepared from stock solutions of $1 \%$ by weight PMMA (75,000 m.w.) in toluene. ADT-TES-F was doped into the solution at the level of $10^{-10}$ per PMMA molecule. This solution was then spun coat onto clean glass coverslips at $2000 \mathrm{rpm}$ for $55 \mathrm{~s}$. The samples were imaged with an Olympus IX71 inverted microscope with a 100X UPlanApo objective under wide-field $532 \mathrm{~nm} \mathrm{cw}$ illumination. The PL was detected by an Andor iXon DU-897 EMCCD camera.

\subsection{Transient photocurrent measurements}

\subsubsection{Optical-pump-terahertz (THz) probe spectroscopy}

A detailed description of the optical-pump - THz-probe experimental setup can be found in Lui \& Hegmann, 2001. Briefly, an amplified Ti:Sapphire laser source (800 nm, $100 \mathrm{fs}, 1 \mathrm{kHz})$ was used to produce optical pump pulses at wavelengths of $400-800 \mathrm{~nm}$ through various wave-mixing schemes and $\mathrm{THz}$ probe pulses generated via optical rectification in a $0.5 \mathrm{~mm}$ thick ZnTe crystal. The samples were mounted on 1-2 mm apertures, and both the $\mathrm{THz}$ probe and optical pump pulses were at normal incidence to the surface of the films ( $a-b$ plane) of the single crystal samples. The room-temperature data were taken in air. For temperature dependence measurements, the samples were mounted in an optical cryostat (sample in vapor). The electric field of the THz pulse transmitted through the samples, $T(t)$, was detected by free-space electro-optic sampling in a 2-mm-thick ZnTe crystal and 
monitored at various delay times $(\Delta t)$ with respect to the optical pump pulse. The range of optical pump fluences was $0.9-1.5 \mathrm{~mJ} / \mathrm{cm}^{2}$. No transient photoconductivity was observed upon optical excitation of the substrates alone. Optical excitation of all thin film and single crystal samples resulted in a change in the transmitted electric field $(-\Delta T(\mathrm{t}))$ due to the transient photoconductivity (i.e. mobile photogenerated carriers) (Hegmann et al., 2002; Hegmann et al., 2005; Ostroverkhova et al., 2005a; Ostroverkhova et al., 2005b; Ostroverkhova et al., 2006a; Ostroverkhova et al., 2006b). In the absence of the phase shift between $\mathrm{THz}$ waveforms obtained in the unexcited and optically excited sample, as was the case for all our samples, the optically induced relative change in the $\mathrm{THz}$ peak amplitude ($\left(T-T_{0}\right) / T_{0} \equiv-\Delta T / T_{0}$, where $T_{0}$ is the amplitude of the $\mathrm{THz}$ pulse transmitted through unexcited sample) provides a direct measure of the transient photoconductivity (Hegmann et al., 2002; Thorsmølle et al., 2004; Ostroverkhova et al., 2005a). The time resolution of this experimental setup was $\sim 400 \mathrm{fs}$. In the approximation of a thin conducting film on an insulating substrate, the differential transmission $\left(-\Delta T / T_{0}\right)$ due to optical excitation of mobile carriers at small $\left|\Delta T / T_{0}\right|$ is related to the transient photoconductivity as follows (Lui and Hegmann, 2001; Hegmann et al., 2002; Thorsmølle et al., 2004):

$$
\sigma=-\left(\Delta T / T_{0}\right)(1+N) /\left(Z_{0} L\right)
$$

where $Z_{0}=377 \Omega$ is the impedance of free space, $N$ is the refractive index of the substrate at $\mathrm{THz}$ frequencies, and $L$ is the film thickness. Using this expression, and setting the maximum value for the transient response at $\Delta t=0$ so that $\left|\Delta T / T_{0}\right|_{\operatorname{MAX}}=\left|\Delta T(0) / T_{0}\right|$, the product of the charge carrier mobility $(\mu)$ and photogeneration efficiency $(\eta)$ can be calculated as follows (Hegmann et al., 2002; Ostroverkhova et al., 2005a; Ostroverkhova et al., 2005b):

$$
\mu \eta=\left|\frac{\Delta T(0)}{T_{0}}\right| \frac{h \nu(1+N)}{e F(1-R)(1-\exp [-\alpha L]) Z_{0}},
$$

where $e$ is the electric charge, $h$ is Planck's constant, $v$ is the light frequency, $\alpha$ is the absorption coefficient, $F$ is the incident fluence, and $R$ is the reflection coefficient.

\subsubsection{Direct measurements of fast photocurrent using digital sampling oscilloscope}

For transient photoconductivity measurements on sub-100 ps to hundreds of microsecond $(\mu \mathrm{s})$ time-scales after pulsed photoexcitation, an amplified Ti:Sapphire laser $(800 \mathrm{~nm}, 100 \mathrm{fs}$, $1 \mathrm{kHz}$ ) was used in conjunction with a frequency-doubling beta-barium borate (BBO) crystal to excite the samples. Voltage was supplied by a Keithley 237 source-measure unit, and light pulse-induced transient photocurrent was measured with a $50 \Omega$ load by a $50 \mathrm{GHz}$ CSA 8200 digital sampling oscilloscope (DSO). The time resolution of this setup was 30-40 ps.

From the peak of the transient photocurrent $\left(\mathrm{I}_{\mathrm{ph}, \max }\right)$, a product of charge carrier mobility $\mu$ and photogeneration efficiency $\eta$ was calculated using

$$
\mu \eta=\mathrm{I}_{\mathrm{ph}, \max } /\left(e \mathrm{~N}_{\mathrm{ph}} E d\right)
$$

where $\mathrm{N}_{\mathrm{ph}}$ is the number density of absorbed photons per pulse, $E$ is the static electric field ( $E=V / L$, where $V$ is the applied voltage and $L$ is the gap between the electrodes), $e$ is the charge of the electron, and $d$ is the channel width. 


\subsection{Dark current and cw photocurrent measurements}

For dark current and cw photocurrent measurements, the samples were embedded in a fixture incorporating a thermoelectric unit for temperature control (range: 285-350 K). The Keithley 237 source-measure unit was used to measure current through the sample in the absence and in the presence of cw photoexcitation with a Nd:YVO 4 laser at $532 \mathrm{~nm}$. The photocurrent was calculated as the difference between the two.

\subsection{Scanning photocurrent microscopy}

Scanning photocurrent microscopy has been previously utilized in probing internal electric field distributions, mapping electronic band structure, measuring mobility-lifetime products, etc. in inorganic and organic films, nanowires, carbon nanotubes, and graphene sheets (Gu et al., 2006; Agostinelli et al., 2007; Ahn et al., 2007; Park et al., 2009). In our experiments, ADT films on coplanar electrodes, either $\mathrm{Al}$ or $\mathrm{Au}$, separated by a $50-\mu \mathrm{m}$ gap, were excited with a focused beam from the glass substrate side, and photoresponse was monitored as the excitation spot was moved across the gap from one electrode to another (Day et al., 2009), under applied voltage. This experiment was performed with either pulsed 100-fs 400-nm excitation or cw 532-nm excitation, using laser sources described above. In the case of pulsed excitation, a lens with $2.5-\mathrm{cm}$ focal distance was used to focus the beam with pulse energy of $\sim 1 \mathrm{~nJ}$ to an approximately $4-\mu \mathrm{m}$ spot at the sample. The lens was translated along the gap using a micrometer-controlled translation stage. At each position of the lens, transient photocurrent resulting from excitation of a localized region of the film was recorded with the DSO. In the case of cw excitation, the sample was placed on an Olympus IX-71 inverted microscope, and a 10x objective with a numerical aperture of 0.6 was used to focus the beam at a power of $\sim 0.4 \mu \mathrm{W}$ to an approximately $400-\mathrm{nm}$ spot. Position of the localized excitation with respect to the electrodes was monitored by a CCD camera that detected fluorescence (emitted by the photoexcited region of the sample) collected through the same objective. The sample was translated using a closed-loop piezoelectrically controlled $x-y$ stage with sub-nm resolution, with a speed of $1 \mu \mathrm{m} / \mathrm{s}$. Cw light was chopped at $565 \mathrm{~Hz}$, and the amplitude of the modulated photocurrent signal was measured by a Stanford Research Systems 830 lock-in amplifier. The experiment was repeated as a function of applied voltage and light power, as well as at different voltage polarities, directions of the scan, and lengths of the waiting period between successive scans.

\section{Results}

\subsection{Optical properties}

\subsubsection{Dilute solutions}

Figure 2 shows optical and PL properties of functionalized ADT and pentacene derivatives in toluene solution. In all spectra, vibronic progression due to coupling of the electronic state to ring-breathing vibrational modes $\left(\sim 1400 \mathrm{~cm}^{-1}\right)$, characteristic of oligoacenes, is observed (Pope \& Swenberg, 1999). In solutions, optical properties were determined primarily by the core of the molecule and were not affected by TIPS or TES side-groups, which resulted in identical spectra of, for example, ADT-TES-F and ADT-TIPS-F in Fig. 2 or TIPS- and TESpentacene (only TIPS-pentacene data are shown). Spectra of ADT-TIPS-CN and TIPSpentacene in solution were both red-shifted with respect to those of ADT-TIPS(TES)-F. Small Stokes shifts of $<10 \mathrm{~nm}$, observed in all solutions, are due to rigidity of the molecular core of oligoacenes. PL lifetime decay of solutions was well described by a single-exponential 
function ( $\exp [-t / \tau]$, where $\tau$ is the PL lifetime). ADT-TIPS-F and ADT-TES-F derivatives exhibited similar lifetimes $(\tau)$ of $\sim 9$ ns and high PL quantum yields (QYs) $(\Phi)$ of $\sim 70 \%$ in toluene. Solutions of ADT-TIPS-CN and TIPS-pentacene showed longer lifetimes ( 12-12.5 ns) and QYs 75\% (Table 2) (Platt et al., 2009a; Platt et al., 2009b; Platt et al., 2009c).
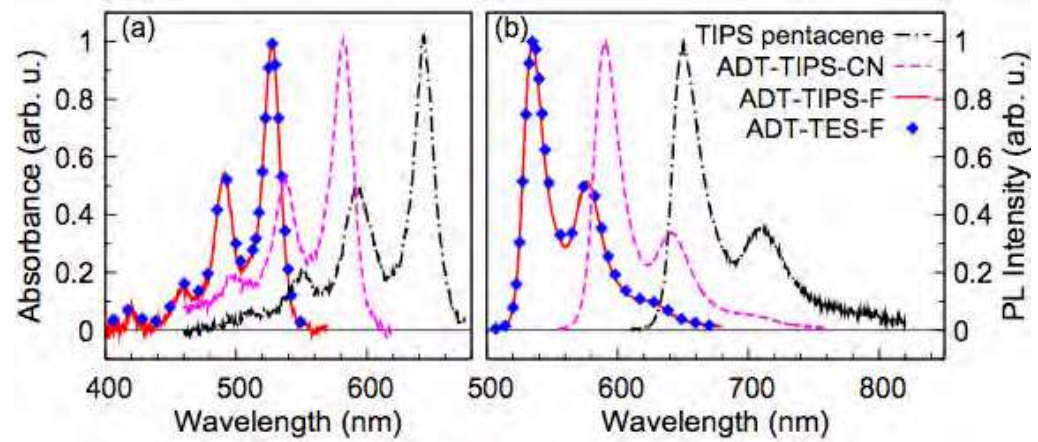

Fig. 2. Normalized optical absorption (a) and PL (b) spectra of functionalized pentacene and ADT derivatives in toluene solution. Spectra of ADT-TES-F and ADT-TIPS-F in solutions are identical. Reprinted from Platt et al., 2009b, with permission. Copyright American Chemical Society (2009).

\subsubsection{Thin films}

Optical absorption spectra of films (Fig. 3(a)) exhibited a redshift, or displacement $\Delta$, with respect to those in solutions, due to enhanced Coulomb interaction of the molecule with its surrounding and exchange interaction between translationally equivalent molecules (Ostroverkhova et al., 2005b). In general, $\Delta$ depends on the molecular-orbital overlap and on the structure and morphology of the film. In contrast to identical absorption and PL spectra of ADT-TIPS-F and ADT-TES-F in solution, those of corresponding films were considerably different (Fig. 3), which we attribute to differences in packing of these molecules in the solid and in film crystallinity. In particular, ADT-TIPS-F and ADT-TES-F films exhibited displacements $\Delta$ of $\sim 320 \mathrm{~cm}^{-1}$ and $\sim 760 \mathrm{~cm}^{-1}$, respectively. Although $\Delta$ exhibited sample-tosample variation, it was always larger in ADT-TES-F films compared to films of ADT-TIPS$\mathrm{F}$, indicative of a higher degree of exciton delocalization in ADT-TES-F films. Also redshifted were PL spectra of ADT-TES-F with respect to those of ADT-TIPS-F films (Fig. 3(b)). Vibronic bands in both absorption and PL spectra were broader in films, as compared to solutions, with relative intensities of the bands varied depending on the film thickness and morphology (Platt et al., 2009a; Platt et al., 2009b). Regardless of the film thickness, ADT-TIPS-F films showed a more pronounced vibronic structure of the PL spectra than ADT-TES-F or ADT-TIPS-CN. The differences observed in PL spectra of films compared to those in solutions are due to intermolecular interactions leading to a formation of crystallites and molecular aggregates (whose properties depend on the degree of molecular order, size, and intermolecular coupling). This is further supported by our observations that the PL spectra of molecules under study embedded at low concentrations in the PMMA matrix yielded spectra identical to those of solutions in Fig. 1(b), as expected from non-interacting molecules (Platt et al., 2009b). Although all materials studied could be prone to aggregate formation due to their $\pi$-stacking properties, PL properties of aggregates significantly 


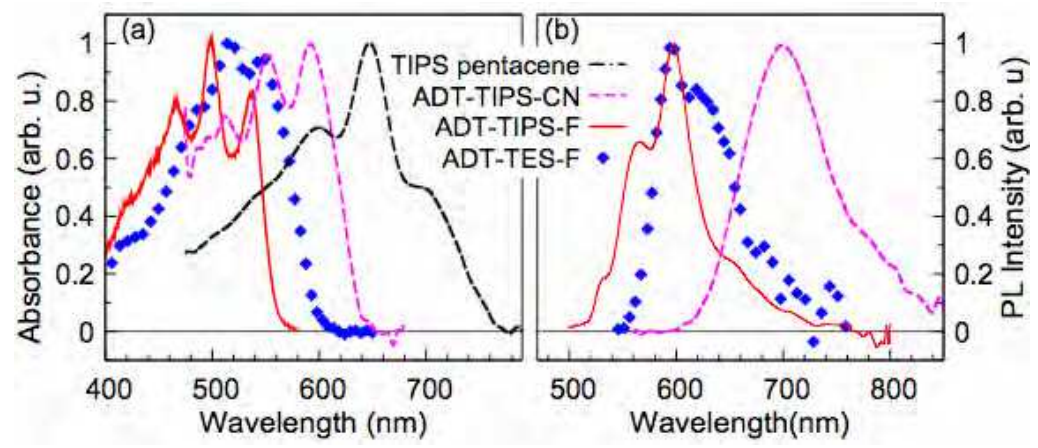

Fig. 3. Normalized optical absorption (a) and PL (b) spectra in TIPS-pentacene and ADT films. No PL response was observed in TIPS-pentacene films. Reprinted from Platt et al., 2009b, with permission. Copyright American Chemical Society (2009).

depended on the material. For example, at room temperature, thin films of ADT-TIPS-F and ADT-TES-F were highly luminescent, with PL QYs reaching $40-50 \%$ depending on the film thickness and morphology. (These values represent a lower limit, since effects of selfabsorption were significant in even the thinnest of our films). In contrast, PL in TIPSpentacene films was so low that it could not be detected (QYs of $<0.5 \%$ ).

In all materials studied, the PL decay dynamics in films were faster than those in solutions and could be described by a bi-exponential function $\left(\sim \mathrm{a}_{1} \exp \left[-t / \tau_{1}\right]+\mathrm{a}_{2} \exp \left[-t / \tau_{2}\right]\right.$, where $\tau_{1(2)}$ and $a_{1(2)}$ are shorter (longer) lifetimes and their relative amplitudes, respectively, and $a_{1}+a_{2}=1$ ), characteristic of molecular aggregates. Both $\tau_{1}$ and $\tau_{2}$ were shorter than lifetimes $\tau$ measured in solutions of the same molecules, and the weighted average lifetimes in films, $\tau_{\mathrm{av}}=\mathrm{a}_{1} \tau_{1}+\mathrm{a}_{2} \tau_{2}$, were typically on the order of $0.4-4 \mathrm{~ns}$ at room temperature, depending on the material, and varied with film quality (e.g. from 1.1 to $2.5 \mathrm{~ns}$ in ADT-TIPS-F films).

Temperature dependence. In all ADT films, the PL response was strongly temperature dependent, and PL quantum yields decreased by a factor of 3-6 as the temperature increased from $5{ }^{\circ} \mathrm{C}$ to $80{ }^{\circ} \mathrm{C}$, depending on the sample. Figure 4 (a) shows (i) PL QY calculated from the integrated PL spectrum measured at $400 \mathrm{~nm}$ excitation, (ii) the peak PL intensity measured under pulsed $80 \mathrm{fs} 400 \mathrm{~nm}$ excitation with the time resolution of $\sim 200$ ps, and (iii) integrated time-resolved fluorescence decay measured under pulsed $80 \mathrm{fs} 400$ nm excitation, all normalized by their values at room temperature of $20^{\circ} \mathrm{C}$, as a function of temperature in an ADT-TIPS-F film. Considerable temperature dependence of the peak PL intensity suggests significant contribution of processes occurring on sub-200 ps time scales, not resolved in our experiments, to the overall temperature dependence of the QYs. The remaining contribution is due to processes occurring on time scales of $<2-4$ ns. Since no temperature dependence of PL emission of our molecules in solution was observed, the strong temperature dependence observed in films is due to temperature-dependent intermolecular interactions in films (Platt et al., 2009b). In order to quantify the observed temperature dependence, we consider PL quantum yield to be inversely proportional to a sum of temperature independent radiative rate and thermally activated non-radiative rate, so that

$$
1 / \Phi \sim 1+a \exp \left[-\Delta_{\mathrm{PL}} / \mathrm{k}_{\mathrm{B}} \mathrm{T}\right]
$$


where $\mathrm{k}_{\mathrm{B}}$ is the Boltzmann constant, $\mathrm{T}$ is temperature, and $a$ is a fitting parameter related to the ratio between radiative and temperature-independent non-radiative rate prefactor. PL quenching activation energies $\Delta_{\mathrm{PL}}$, obtained from fits of data to Eq.(4) yielded values between 0.11 and $0.27 \mathrm{eV}$, depending on the sample and on the material (e.g. $0.15 \mathrm{eV}$ in an ADT-TIPS-CN film in Fig.4(b)) (Platt et al., 2009b).
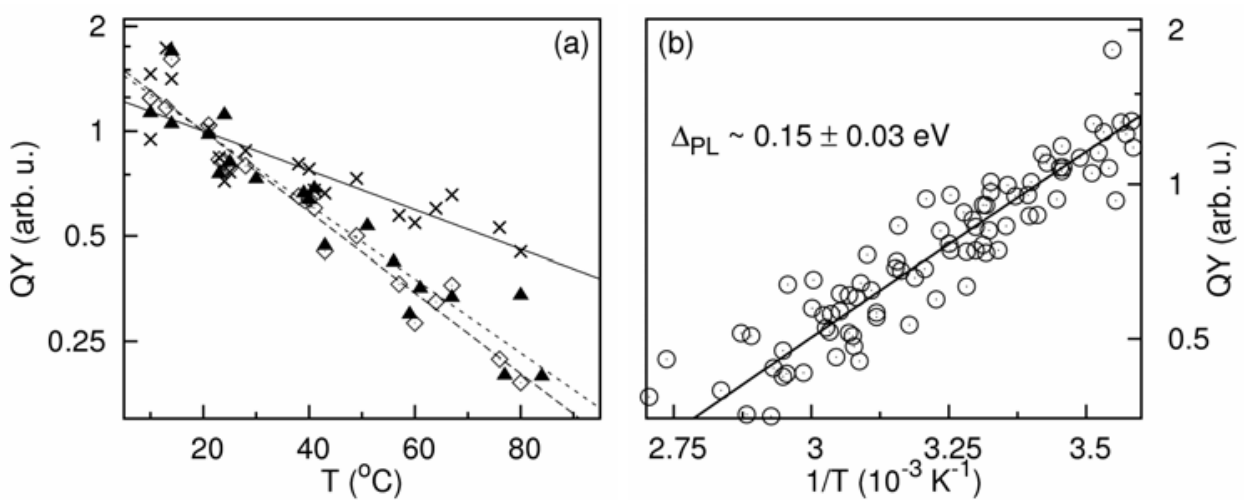

Fig. 4. (a) Temperature dependence of (i) the QYs calculated from the integrated PL spectrum measured at $400 \mathrm{~nm}$ excitation (triangles), (ii) peak amplitude of the PL transient measured under pulsed $80 \mathrm{fs} 400 \mathrm{~nm}$ excitation with 200 ps resolution ( $\times$ ), and (iii) integrated PL decays measured under pulsed $80 \mathrm{fs} 400 \mathrm{~nm}$ excitation (diamonds) in ADTTES-F film. All are normalized by their values at room temperature of $20^{\circ} \mathrm{C}$.

(b) Temperature dependence of the QY, normalized at its value at room temperature, obtaine in an ADT-TIPS-CN film. The fit of the QY by Eq. (4) is also shown. Reprinted from Platt et al., 2009b, with permission. Copyright American Chemical Society (2009).

\begin{tabular}{|c|c|c|c|c|c|c|c|c|}
\hline Compound & $\begin{array}{c}\mathrm{HOMO}^{\mathrm{a}} \\
(\mathrm{eV})\end{array}$ & $\begin{array}{c}\mathrm{LUMO}^{\mathrm{a}} \\
(\mathrm{eV})\end{array}$ & $\begin{array}{c}\lambda_{\mathrm{abs}^{\mathrm{b}}} \\
(\mathrm{nm})\end{array}$ & $\lambda_{\mathrm{PL}^{\mathrm{b}}}(\mathrm{nm})$ & $\Phi \mathrm{c}$ & $\tau \mathrm{c}(\mathrm{ns})$ & $\begin{array}{c}\lambda_{\mathrm{abs}_{\text {,film }}{ }^{\mathrm{d}}} \\
(\mathrm{nm})\end{array}$ & $\begin{array}{c}\lambda_{\mathrm{PL}, \text { film }}{ }^{\mathrm{d}} \\
(\mathrm{nm})\end{array}$ \\
\hline ADT-TES-F & -5.35 & -3.05 & 528 & 536 & 0.70 & 9.4 & 550 & 595 \\
\hline ADT-TIPS-F & -5.35 & -3.05 & 528 & 536 & 0.72 & 9.1 & 537 & 600 \\
\hline ADT-TIPS-CN & -5.55 & -3.49 & 582 & 590 & 0.76 & 12.7 & 592 & $\sim 688$ \\
\hline TIPS-pent. & -5.16 & -3.35 & 643 & 650 & 0.75 & 11.8 & $\sim 705$ & -- \\
\hline
\end{tabular}

Table 2. Electrochemical and photophysical properties of TIPS-pentacene and ADT derivatives.

a Measured using differential pulse voltammetry (Platt et al., 2009b)

$\mathrm{b}$ Wavelengths of maximal absorption or PL corresponding to $0 \rightarrow 0$ transitions in toluene solutions

c PL QY and PL lifetime in toluene solutions

$\mathrm{d}$ Wavelengths of maximal absorption or PL in thin films. PL from TIPS-pentacene films could not be detected (QY<0.5\%)

Electric field dependence. In order to address a possibility of electric field-induced dissociation of the radiative state, we measured PL spectra and lifetimes upon either cw 532 
nm or pulsed $80 \mathrm{fs} 400 \mathrm{~nm}$ photoexcitation of ADT films, under applied voltage. No changes in the PL QYs, peak PL intensity, or PL lifetimes have been detected at the applied voltages of $0-500 \mathrm{~V}$, i.e., up to average electric fields of $2 \times 10^{5} \mathrm{~V} / \mathrm{cm}$, at any temperature in the range of $5-80{ }^{\circ} \mathrm{C}$.

\subsubsection{Composite thin films}

Figure 5 shows absorption and PL spectra of the composite films containing ADT-TES-F host molecules and $2 \mathrm{wt} \% \mathrm{C}_{60}, 10 \mathrm{wt} \%$ of TIPS-pentacene or $10 \mathrm{wt} \%$ of ADT-TIPS-CN guest molecules. Based on the absorption spectra in Fig. 5(a)), no ground state charge transfer occurs upon addition of guest molecules at these concentrations. In contrast to absorption spectra, addition of any guest molecules to ADT-TES-F in film produced a dramatic effect on the PL spectra (Fig. 5(b)), due to photoinduced charge or energy transfer, depending on the guest (Day et al., 2009a). In particular, very strong photoinduced energy transfer was observed in ADT-TES-F/ADT-TIPS-CN composites: even at a concentration of ADT-TIPS$\mathrm{CN}$ in ADT-TES-F as low as $0.1 \%$, almost no PL emission was observed from the ADT-TES-F host, and the PL response of the film was dominated by that of the ADT-TIPS-CN guest molecules (Platt et al., 2009c). In contrast, photoinduced charge transfer, leading to the PL quenching, is more favored in ADT-TES-F/ $\mathrm{C}_{60}$ and ADT-TES-F/TIPS-pentacene composites. As we discuss in Section 3.3.2, PL properties of the composites are correlated with photoconductive properties of the same samples on short time-scales after a pulsed $80 \mathrm{fs}$ photoexcitation.

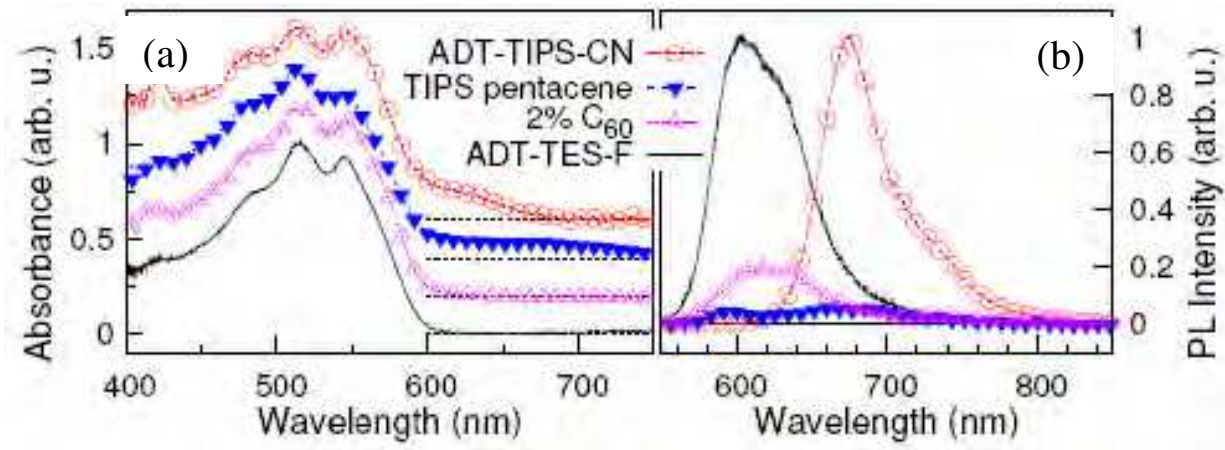

Fig. 5. Optical absorption (a) and PL (b) spectra of a pristine ADT-TES-F film and composites ADT-TES-F/C 60 (2\%), ADT-TES-F/TIPS-pentacene (10\%), and ADT-TESF/ADT-TIPS-CN (10\%). Absorption spectra are shifted along y-axis for clarity. Reprinted from Day et al., 2009a, with permission. Copyright American Institute of Physics (2009).

\subsection{Properties of individual molecules}

Figure 6 shows a typical time trajectory of the PL photon count obtained from an individual ADT-TES-F molecule in a PMMA matrix, as confirmed by a single-step photobleaching at time $t=32 \mathrm{~s}$. This demonstrates that PL quantum yields and photostablity of ADT-TES-F molecules are good enough to enable imaging at the single molecule level at room temperature. Signal-to-noise ratios of over 20:1 were obtained at moderate excitation levels at $532 \mathrm{~nm}$ in the epi-illumination geometry. Moreover, ADT-TES-F exhibited remarkably good stability as a single molecule fluorophore, comparable to DCDHF-N-6, which is 
currently one of the best fluorophores utilized in single-molecule fluorescence spectroscopy. This opens up new possibilities to study charge- and energy-transfer processes in these materials at nanoscales, which are currently underway in our laboratory.

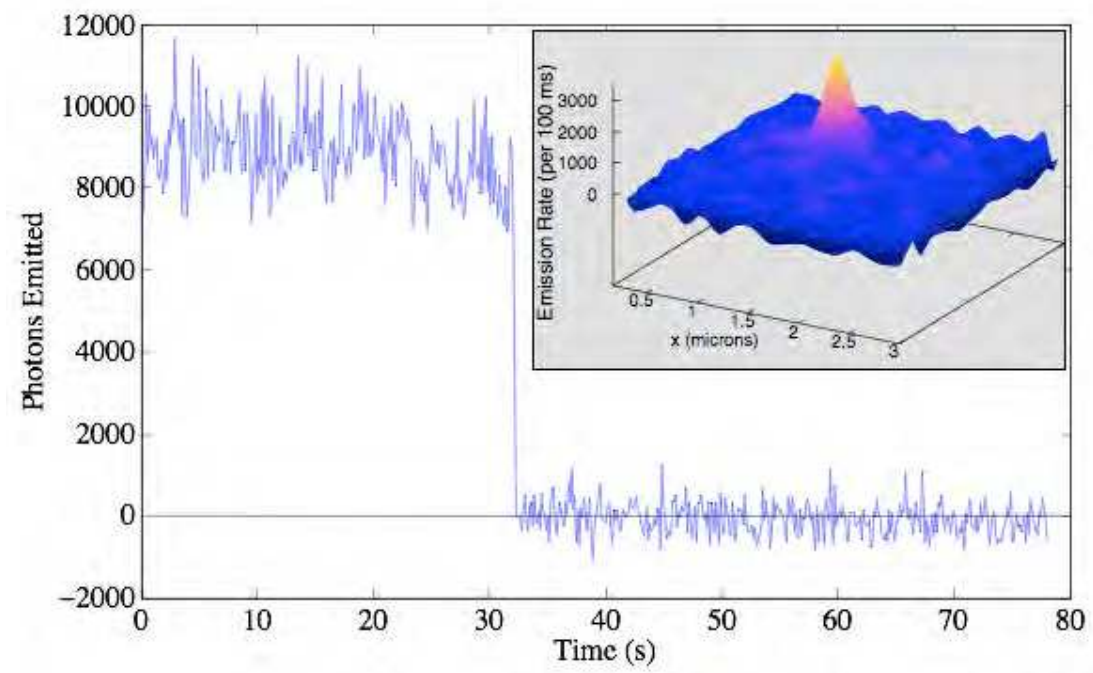

Fig. 6. Time trace of the fluorescence of a single molecule of ADT-TES-F under $700 \mu \mathrm{W}$ widefield $532 \mathrm{~nm}$ cw illumination. Single-step photobleaching at $\sim 32 \mathrm{~s}$ confirms that the trace belongs to a single-molecule. Inset shows a surface plot of the emission of the same molecule with cubic interpolation. Integration time was $100 \mathrm{~ms}$.

\subsection{Transient photoconductivity}

\subsubsection{Non-contract measurements on $400 \mathrm{fs}-1$ ns time-scales using optical pump-THz probe spectroscopy}

Figure $7(\mathrm{a})$ illustrates the differential $\mathrm{THz}$ transmission $\left(-\Delta T / T_{0}\right)$ under optical excitation at $580 \mathrm{~nm}$ as a function of delay time $(\Delta t)$ between the optical pump and THz probe pulses, obtained in Pc, TIPS-pentacene, Rub and Tc crystals and Pc and TIPS-pentacene thin films (Ostroverkhova et al., 2005a; Ostroverkhova et al., 2005b; Ostroverkhova et al., 2006a). All transients, except that for the Tc, which was taken at $10 \mathrm{~K}$, were measured at room temperature in air. The onset of the photoresponse reveals a fast photogeneration process for mobile carriers with characteristic times below $\sim 400 \mathrm{fs}$ limited by the time resolution of our setup in all samples. This suggests that free carriers, and not only excitons, can be created in these materials fast, and in the absence of applied electric field. At room temperature (RT), measurements of the peak value of $-\Delta T / T_{0}$ yielded $\mu \eta$ values (calculated from Eq.(2)) of $\sim 0.3-0.35,0.15-0.2,0.05-0.06$, and $0.03 \mathrm{~cm}^{2} /(\mathrm{Vs})$ for Pc, TIPS-pentacene, Rub and Tc crystals, respectively, and 0.02-0.03 and 0.01-0.06 in Pc and TIPS-pentacene thin films, depending on their structure. These values (in particular the photogeneration efficiency $\eta$ ) include initial carrier trapping and recombination occurring within $400 \mathrm{fs}$ after photoexcitation, not resolved in our experiment. If we assume $\eta=1$, then these $\mu \eta$ values provide a lower estimate for the carrier mobility $\mu$. Since $\eta<1$, the mobility value is higher. 
Likewise, if we assume that RT intrinsic mobilities in these materials are on the order of $<10$ $\mathrm{cm}^{2} / \mathrm{Vs}$ in TIPS-pentacene and Tc and $\sim 20-30 \mathrm{~cm}^{2} /(\mathrm{Vs})$ in Rub and Pc (Jurchescu et al., 2004; Podzorov et al., 2004), then the lower limit for the photogeneration efficiency in single crystals is $\sim 1-2 \%$ in Pc and TIPS-pentacene and $\sim 0.3 \%$ in Rub and Tc. The observed difference in $\mu \eta$ could be due to the differences in: (i) intrinsic carrier mobility, (ii) initial photogenerated free carrier yield, and (iii) carrier loss due to charge trapping and recombination. Factor (iii) is likely not to be a significant contributor at such short timescales after photoexcitation. This is supported by our observation of the $\mu \eta$ values measured in good-quality TIPS-pentacene thin films reaching $30-40 \%$ of those obtained in TIPSpentacene single crystals, in spite of the abundance of deep traps at the grain boundaries in thin films (Ostroverkhova et al., 2005b). Therefore, the differences in the trapping properties of TIPS-pentacene, Pc, Rub and Tc crystals most likely account only for a factor up to $\sim 2$ in the differences in $\mu \eta$ values obtained in these crystals at RT, while further differences are due to factors (i) and (ii). For example, the lower transient photoconductivity observed in Rub compared to Pc single crystals at RT at $400 \mathrm{~nm}$ could be mostly due to the lower photogeneration efficiency $\eta$ (factor (ii)) in Rub, in qualitative agreement with Podzorov et al., 2004 and Lang et al., 2004.

(a)

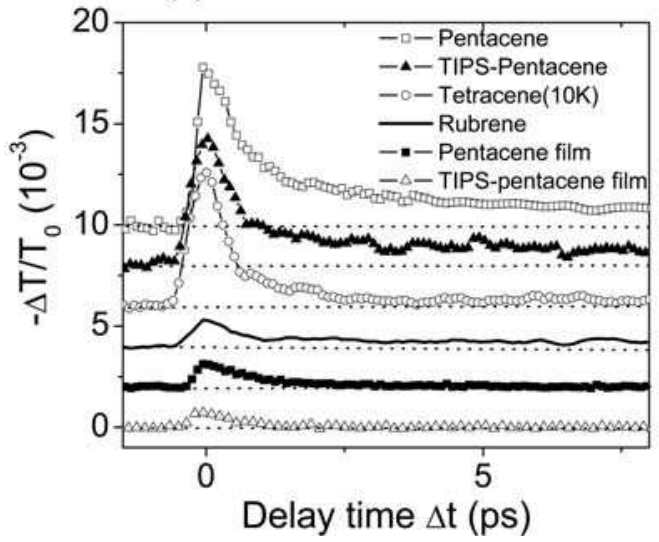

(b)

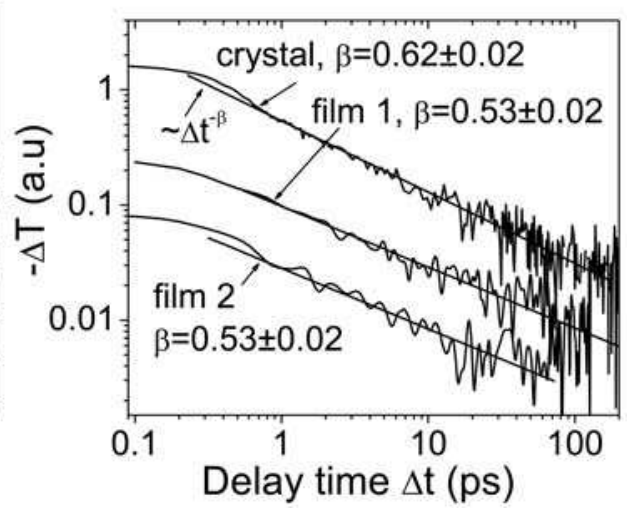

Fig. 7. (a) Differential THz transmission due to transient photoconductivity in various organic crystals and thin films as a function of pump-probe delay time. (b) Decay dynamics of the transient photoconductivity in TIPS-pentacene crystal and two films. Fits with a power-law function are also shown. Adapted from Ostroverkhova et al., 2005b and Ostroverkhova et al., 2006a, with permission. Copyright American Institute of Physics (2005, 2006).

The decay dynamics of the transient photoconductivity yield information about the nature of charge transport, trapping and recombination (Etemad et al., 1981; Moses et al., 1987; Yu et al., 1990). In TIPS-pentacene crystals, the transient photoconductivity exhibited a fast initial decay during the first few picoseconds, followed by a slow decay best described by a power law function $\left(\sim \Delta t^{-\beta}\right)$ with $\beta=0.5-0.7$ over many orders of magnitude in time, which has been attributed to dispersive transport (Fig.7(b)) (Etemad et al., 1981; Hegmann et al., 2002). Interestingly, these dynamics in TIPS-pentacene crystals did not change appreciably 
over a wide temperature range of 5-300 K, which suggests tunneling, rather than thermallyactivated hopping, mechanism of charge transport. Similar decay dynamics was observed in Pc crystals at RT and in best TIPS-pentacene films (Ostroverkhova et al., 2005b; Ostroverkhova et al., 2006a). In contrast, photoconductivity in lower-quality TIPS-pentacene and Pc films exhibited fast bi- or single-exponential decay dynamics ( $\left.\exp \left[-\Delta t / \tau_{c}\right]\right)$ with time constants $\tau_{\mathrm{c}} \sim 1$ ps for both materials, independent of the temperature, most likely due to deep-level traps at the interfaces between crystallites.

Wavelength dependence. In order to gain insight into the photogeneration process, we repeated the optical pump-THz probe experiment at various pump wavelengths in TIPSpentacene crystals and Pc and TIPS-pentacene thin films. Due to the short temporal width of the optical pulses $(\sim 100 \mathrm{fs})$, the illumination is not monochromatic, as illustrated in Fig. 8(a), which shows the spectra of some of the optical pump pulses utilized in this experiment. Typical optical absorption spectra of the thin film and single crystal samples are shown in Fig. 8(b). No transient photoresponse was obtained from TIPS-pentacene and Pc thin films upon excitation at $800 \mathrm{~nm}$ - the spectral region in which there is too little absorption (Fig. $8(b))$. At all wavelengths of optical excitation within the absorption spectra, we observed a photoinduced change in $\mathrm{THz}$ transmission with a fast onset and decay dynamics similar to that shown in Fig. 7 in all our samples.

Furthermore, $\mu \eta$ calculated from our data was wavelength-independent within our experimental error, as shown in Fig. 8(c). Wavelength-independent photocarrier generation has also been observed in other organic semiconductors using ultrafast techniques (Moses et al., 2000). However, conventional steady-state photoconductivity measurements typically exhibit wavelength-dependent mobile carrier photogeneration efficiencies (Silinsh \& Capec, 1994; Pope \& Swenberg, 1999), as reported for pentacene single crystals (Lang et al., 2004).

Temperature dependence. The temperature dependence of the photoresponse provides valuable information about the mechanism of photoconductivity (Moses, 1989; Karl, 2001; Karl, 2003). In both single crystals and thin films, the transient photoconductivity increased as the temperature decreased, as demonstrated in Fig. 8(d). As the temperature decreased from $297 \mathrm{~K}$ to about $20 \mathrm{~K}, \mu \eta$ increased in all crystals by a factor of 3-10, depending on the crystal. Assuming $\eta$ does not increase as the temperature decreases, we can attribute the temperature dependence of $\mu \eta$ shown in Fig. 8(d) to band-like charge carrier transport (Hegmann et al., 2002; Thorsmølle et al., 2004; Ostroverkhova et al., 2005a; Ostroverkhova et al., 2005b), which has not been previously observed over such a wide temperature range in Tc and Rub single crystals (de Boer et al., 2004; Newman et al., 2004; Podzorov et al., 2004). The initial increase of $\mu \eta$ by a factor of 1.5 - 3 (depending on the crystal) as the temperature is lowered from $297 \mathrm{~K}$ to about $150 \mathrm{~K}$ (Fig.8(d)) is consistent with the increase in charge carrier mobility observed from field-effect measurements in Rub (Podzorov et al., 2004) and from space-charge-limited-current measurements in Pc (Jurchescu et al., 2004) and Tc (de Boer et al., 2004) single crystals over the same temperature range. In films, the $\mu \eta$ increased from $\sim 0.02 \mathrm{~cm}^{2} /(\mathrm{Vs})$ to $\sim 0.07 \mathrm{~cm}^{2} /(\mathrm{Vs})$ as the temperature decreased from $297 \mathrm{~K}$ to $5 \mathrm{~K}$, the trend which has not been previously observed in polyacene thin films. In addition, even though such band-like mobility has been obtained in several organic crystals (Moses, 1989; Karl, 2001; Hegmann et al., 2002; Karl, 2003; de Boer et al., 2004; Jurchescu et al., 2004; Podzorov et al., 2004; Thorsmølle et al., 2004), only a few studies reported the band-like behavior over a wide temperature range (Karl, 2001; Hegmann et al., 2002; Karl, 2003; Thorsmølle et al., 2004), as observed in our experiments (Fig.8). The thin film data in Fig. 
8 (d) can be fitted with the power law function $\mu \eta \sim \mathrm{T}^{-\mathrm{n}}$, with the exponent $\mathrm{n} \sim 0.32$, similar to $\mathrm{n} \sim 0.27$ reported from transient photoconductivity measurements of Pc single crystals. (Thorsmølle et al., 2004).

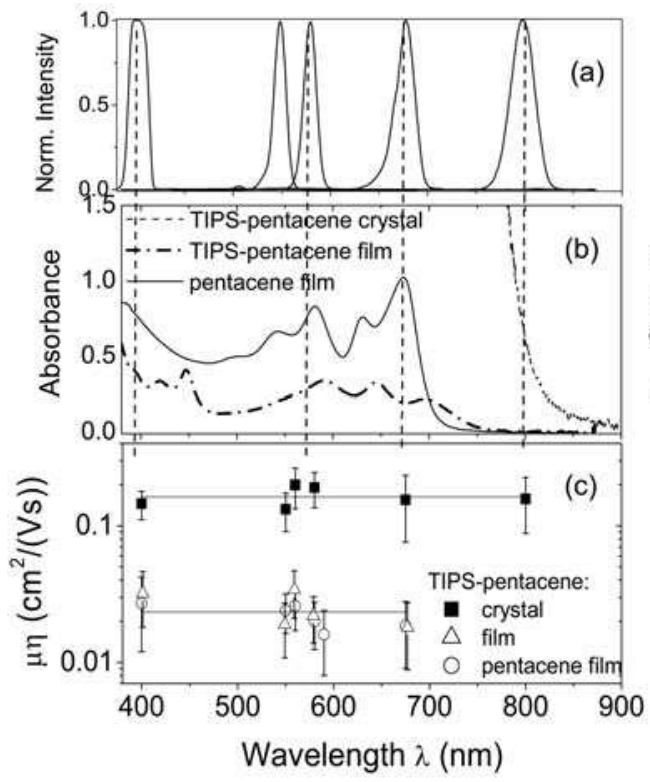

(d)

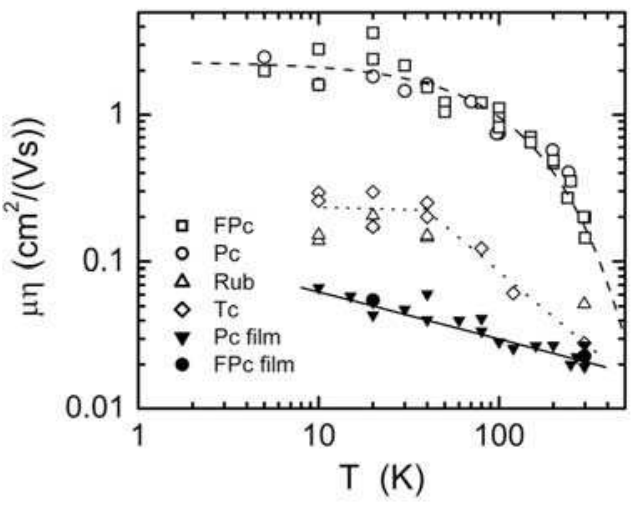

Fig. 8. (a) Normalized intensity spectra of selected optical pump pulses. Spectra of pulses centered at 550 and $590 \mathrm{~nm}$ are not included for clarity. (b) Absorption spectra of a TIPSpentacene crystal and TIPS-pentacene and Pc thin-film samples. (c) Product of mobility and photogeneration efficiency $(\mu \eta)$ measured in a TIPS-pentacene crystal and TIPS-pentacene and Pc thin-film samples at room temperature. (d) Temperature dependence of the $\mu \eta$ obtained in Pc, Rub, Tc, and TIPS-pentacene (the latter labeled as FPc on the figure) single crystals and Pc and TIPS-pentacene (labeled as FPc) films. Adapted from Ostroverkhova et al., 2005a and Ostroverkhova et al., 2006a, with permission. Copyright American Physical Society (2005) and American Institute of Physics (2006).

Photoconductivity anisotropy. In order to probe the effect of molecular packing on the transient photoconductivity, we performed optical pump-THz probe experiments in TIPSpentacene and TES-pentacene single crystals (Fig.9(a)-(c)) (Ostroverkhova et al., 2006b). The $-\Delta T / T_{0}$ transients observed in both TIPS-pentacene and TES-pentacene crystals had very similar shapes and exhibited sub-picosecond charge photogeneration and power-law decay dynamics discussed above (Fig.7). The $\mu \eta$ product, calculated using Eq.(2) from the peak of the $-\Delta T / T_{0}$ transient obtained with both the electric field of the $\mathrm{THz}$ probe pulse $\left(\mathrm{E}_{\mathrm{THz}}\right)$ and that of the optical pump pulse (E $\mathrm{E}_{\text {pump}}$ ) parallel to the $a$-axis, yielded $\sim 0.15-0.2 \mathrm{~cm}^{2} /(\mathrm{Vs})$ and $\sim 0.05-0.06 \mathrm{~cm}^{2} /(\mathrm{Vs})$ for the charge carrier mobility in TIPS-pentacene and TES-pentacene crystals, respectively, depending on the sample. As discussed above, these numbers represent lower limits for the charge carrier mobility along the $a$-axis. The triclinic symmetry group of TIPS-pentacene and TES-pentacene crystals (Table 1) leads to a complex picture of charge transport described by six components of the mobility tensor $\mu_{\mathrm{ij}}$, where $\mathrm{i}, \mathrm{j}=x, y, z$, 
are components in an orthogonal coordinate system (choice of which is somewhat arbitrary), and $\mu_{\mathrm{ij}}=\mu_{\mathrm{ji}}$. In general, the principal axes of the mobility tensor do not coincide with the crystallographic axes, are different for hole and electron transport, and are temperaturedependent (Karl, 2001). In order to measure charge carrier mobility anisotropy, separately from that of the photogeneration efficiency, we performed the following experiments (Ostroverkhova et al., 2006b). The crystal was rotated in the azimuthal $(a-b)$ plane, so that the angle $(\varphi)$ between the direction of $\mathrm{E}_{\mathrm{THz}}$ and the $a$-axis of the crystal changed (at $\varphi=0^{\circ}$ $\mathrm{E}_{\mathrm{THz}}$ was parallel to the $a$-axis). The polarization of the pump ( $\left.\mathrm{E}_{\text {pump }}\right)$ was rotated with the crystal using a half-wave plate and a polarizer combination in order to maintain $\mathrm{E}_{\text {pump }}$ parallel to the $a$-axis and ensure the same fluence at all angles. At every angle, the peak value of $-\Delta T / T_{0}$ was measured and the $\mu \eta$ product was calculated using Eq.(2). In this case, the observed angular dependence of the photoconductivity was purely due to that of the charge carrier mobility, and not due to the combined effects of the mobility and photogeneration efficiency anisotropy. Figures 9 (d) and (e) show the angular dependence of the charge carrier mobility (averaged over all samples measured at 800-nm, 400-nm and 580nm-excitation) normalized to the value at $\varphi=0^{\circ}$ in TIPS-pentacene and TES-pentacene crystals, respectively. The charge carrier mobility along a certain direction $l=\left(l_{\mathrm{i}}, l_{\mathrm{j}}, l_{\mathrm{k}}\right)$ is given by $\mu_{l l}=\mu_{\mathrm{ij}} \mathrm{l}_{\mathrm{i}} \mathrm{l}_{\mathrm{j}}$. For convenience, we chose the orthogonal coordinate system $(x, y, z)$ so that $x|| a$-axis, $y \perp x$ and is in the $a$ - $b$ plane, and $z \perp a-b$ plane. Then, in the $a-b$ plane, $l=$ $(\cos \varphi, \sin \varphi, 0)$, and the mobility along $l$ is:

$$
\mu_{l l}=\mu_{\mathrm{xx}}(\cos \varphi)^{2}+\mu_{\mathrm{yy}}(\sin \varphi)^{2}+\mu_{\mathrm{xy}} \sin (2 \varphi)
$$

For example, the mobility along the $a$-axis is $\mu_{a a}=\mu_{\mathrm{xx}}$. The equation above normalized by $\mu_{\mathrm{xx}}$ was used to fit the data in Fig. 9 (d) and (e) and yielded $\mu_{\mathrm{yy}} / \mu_{\mathrm{xx}}=0.34 \pm 0.05(0.09 \pm 0.05)$ and $\mu_{\mathrm{xy}} / \mu_{\mathrm{xx}}=-0.17 \pm 0.04(0.06 \pm 0.04)$ in TIPS-pentacene (TES-pentacene). Using these components and substituting $\varphi=\gamma$ from Table 1 (for $\mu_{b b}$ ) in Eq.(5), we obtained the ratio of the mobilities along $a$ - and $b$-axis $\left(\mu_{a a} / \mu_{b b}=3.2\right.$ and 8 in TIPS-pentacene and TES-pentacene, respectively). Assuming that $\mu_{\mathrm{yz}}$ and $\mu_{\mathrm{xz}}$ components of the mobility tensor are small, we diagonalized the $x-y$ part of the tensor and determine the directions of the principal axes 1 and 2 , as well as the corresponding mobilities $\mu_{11}\left(1.04 \mu_{a a}\right.$ and $1.004 \mu_{a a}$ in TIPS-pentacene and TES-pentacene, respectively) and $\mu_{22}\left(0.30 \mu_{a a}\right.$ and $0.086 \mu_{a a}$ in TIPS-pentacene and TESpentacene, respectively), whose ratio $\mu_{22} / \mu_{11}$ yielded $3.5 \pm 0.6$ and $12 \pm 6$ for TIPS-pentacene and TES-pentacene, respectively. The mobility anisotropy of 3.5 in the TIPS-pentacene crystal is very similar to that obtained in Rub and Pc single crystals using a field-effect transistor geometry (Podzorov et al., 2004). Considerable difference in the in-plane mobility anisotropy between the TIPS-pentacene and TES-pentacene crystals supports a theoretical prediction of much stronger mobility anisotropy in the case of the TES-pentacene-type crystals that favor 1D charge transport based on the crystal structure and molecular packing (Figs.9(a) and (b)). In TIPS-pentacene, the principal axes 1 and 2 constitute angles $\varphi_{1}=-14^{\circ}$ and $\varphi_{2}=76^{\circ}$ with respect to the a-axis, respectively (Fig.9(d)). Interestingly, the highest mobility axis does not exactly coincide with the direction of maximum $\pi$-overlap along the $a$-axis, which highlights the contribution of factors unrelated to band structure, such as fluctuations of the intermolecular coupling, to charge carrier mobility in organic molecular crystals. In TES-pentacene, the principal (highest mobility) axis 1 is coincident with the direction of maximum $\pi$-overlap along the $a$-axis (Figs. 9(b) and (e)). However, it is possible 
that due to the above mentioned fluctuations in intermolecular electronic coupling, the observed in-plane anisotropy of $\sim 12$ is smaller than that expected based solely on the band structure. The observed mobility anisotropy in these samples, which is one of the signatures of band-like charge transport in organic crystals, further supports the occurrence of bandlike transport in functionalized pentacene single crystals.

(a)
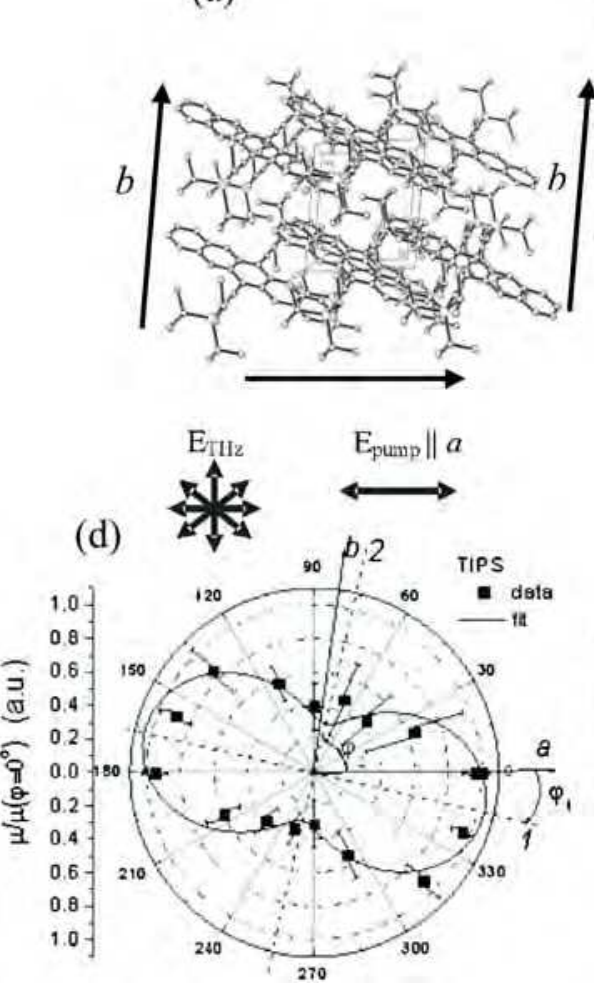

(b)

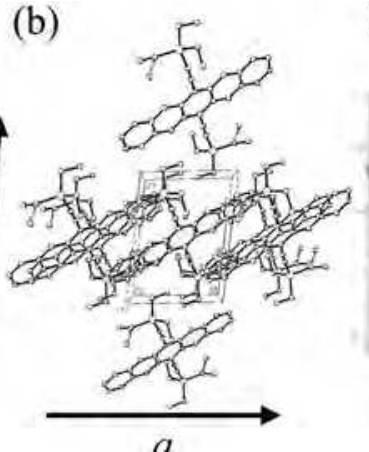

$a$ (c)

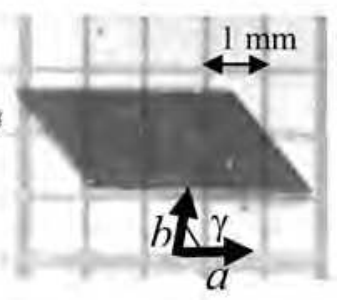

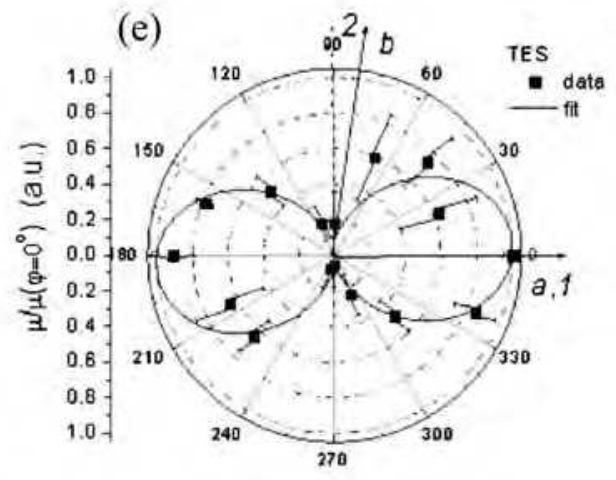

Fig. 9. Molecular packing in (a) TIPS-pentacene and (b) TES-pentacene crystals. (c) Typical TIPS-pentacene crystal. Dependence of the charge carrier mobility on the azimuthal angle $\varphi$ obtained in (d) TIPS-pentacene and (e) TES-pentacene crystals. In both crystals, $\varphi=0^{\circ}$ corresponds to the $a$ axis. Lines correspond to the fit with a function described in the text. Crystallographic $a$ and $b$ axes as well as the principal axes 1 and 2 are also shown. Adapted from Ostroverkhova et al., 2006b, with permission. Copyright American Institute of Physics (2006).

\subsubsection{Transient photocurrent on $\mathbf{3 0}$ ps to $1 \mathrm{~ms}$ time-scales in thin-film device structures}

Pristine Materials. Upon excitation with $400 \mathrm{~nm} 100 \mathrm{fs}$ pulses under applied voltage, thinfilm devices based on TIPS-pentacene and ADT derivatives (ADT-TES-F and ADT-TIPS-F, Section 3.1) showed fast photoresponse, with the rise time of 30-40 ps, limited by the time resolution of the sample fixture and DSO, which supports our earlier observations (using noncontact optical pump-THz probe techniques, Section 3.3.1) of fast charge carrier 
photogeneration in acenes. Transient photocurrents obtained in ADT-TIPS-F and TIPSpentacene films upon excitation with $400 \mathrm{~nm}$ pulses at the electric field $E$ of $1.2 \times 10^{4} \mathrm{~V} / \mathrm{cm}$ are shown in Fig. 10. In all films, transient photocurrents exhibited fast initial decay, most likely due to initial carrier trapping and recombination, followed by a slow component that can be fitted with a power-law function $\left(\mathrm{I}_{\mathrm{ph}} \sim \mathrm{t}^{-\beta}\right)$ with $\beta \sim 0.2-0.6$, depending on the sample and on the material, over at least three orders of magnitude (inset of Fig. 10) (Day et al., 2008; Day et al., 2009a; Platt et al., 2009b). Among ADT-TIPS-F, ADT-TES-F, and TIPSpentacene films, the decay of the transient photocurrent was, on average, slowest in ADTTES-F, followed by ADT-TIPS-F and TIPS-pentacene, when measured under the same conditions. Power-law exponents $\beta$ describing the transient photocurrent decay dynamics on time scales from sub-ns to at least tens of $\mu$ s after photoexcitation were also slightly different, with $\beta$ of $\sim 0.2-0.3$ in ADT-TES-F and of $\sim 0.4-0.6$ in ADT-TIPS-F and TIPSpentacene films. Interestingly, the power-law exponents observed in these experiments in TIPS-pentacene films were similar to those obtained at shorter time-scales in the optical pump-THz probe experiments in similar films and TIPS-pentacene single crystals (Fig.7(b)) (Ostroverkhova et al., 2005a; Ostroverkhova et al., 2005b), which suggests that the same physical mechanism is responsible for charge carrier dynamics observed on time-scales from ps to at least tens of $\mu$ s after photoexcitation.

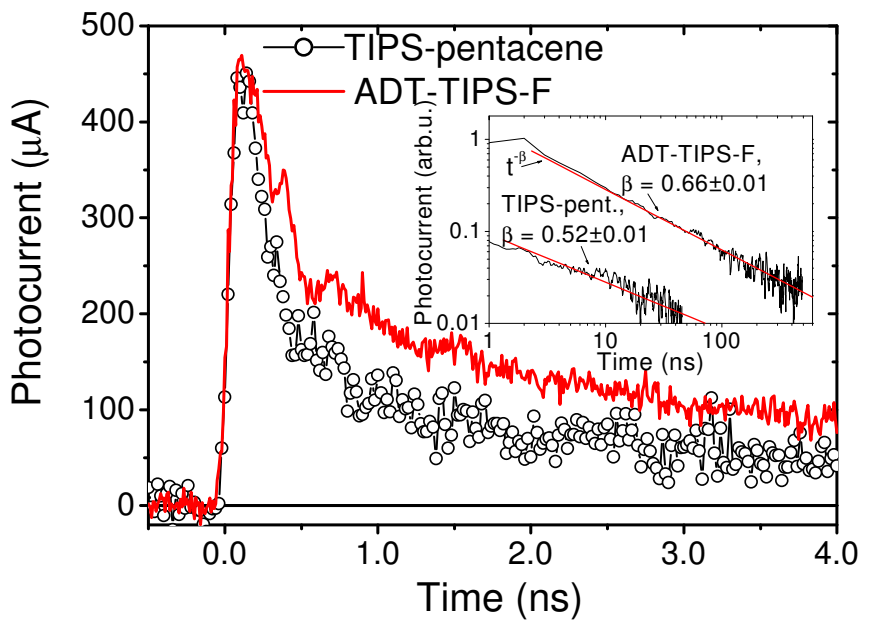

Fig. 10. Transient photocurrent obtained in ADT-TIPS-F and TIPS-pentacene films on Al interdigitated electrodes at $1.2 \times 10^{4} \mathrm{~V} / \mathrm{cm}$ and $30 \mu \mathrm{J} / \mathrm{cm}^{2}$ at $400 \mathrm{~nm}$. Inset: normalized transient photocurrents $\left(I_{\mathrm{ph}}\right)$ in ADT-TIPS-F and TIPS-pentacene films, at longer time-scales. Power-law fits $\left(I_{\mathrm{ph}} \sim t^{-\beta}\right)$ are also shown. Offset along the $y$ axis is for clarity. Adapted from Day et al., 2008, with permission. Copyright American Institute of Physics (2008).

The $\mu \eta$ products calculated from Eq.(3) yielded values between 0.01 and $0.025 \mathrm{~cm}^{2} / \mathrm{Vs}$ at $1.2 \times 10^{4} \mathrm{~V} / \mathrm{cm}$ in ADT-TES-F, ADT-TIPS-F and TIPS-pentacene films (Table 3). Here $\eta$ includes trapping and recombination that occurred during the first tens of picoseconds after 
photoexcitation, not resolved in these experiments, and therefore, $\eta<\eta_{0}$, where $\eta_{0}$ is the initial photogeneration efficiency. If intrinsic mobilities in ADT materials are on the order of $1 \mathrm{~cm}^{2} / \mathrm{Vs}$, then the lower limit for the initial photogeneration efficiency $\eta_{0}$ is $\sim 1-2 \%$, which is comparable to that of $5 \%$ estimated from the transient photocurrents measured in tetracene single crystals under similar conditions (Moses et al., 2006). Similarly to the results of the THz spectroscopy (Section 3.3.1, Fig.8(b)), the $\mu \eta$ obtained from the transient photocurrent amplitudes in these experiments decreased as the temperature increased, at least in the temperature range between $285 \mathrm{~K}$ and $350 \mathrm{~K}$ (Day et al., 2008).

Composites. Both amplitude and decay dynamics of the transient photocurrent can be manipulated by addition of guest molecules in the host material (Section 3.1). Inset of Figure 11 shows peak amplitude of the transient photocurrent as a function of concentration of $\mathrm{C}_{60}$ in the ADT-TES-F/ $\mathrm{C}_{60}$ composite thin film, at 40, 70, and $100 \mathrm{~V}$ (Day et al., 2009a). Addition of $\mathrm{C}_{60}$ at a concentration of 2 and $5 \%$ increased the photocurrent amplitude by a factor of $\sim 3$ and 10, respectively, at $40 \mathrm{~V}$. This increase is most likely due to fast photoinduced electron transfer (a mechanism similar to that leading to sensitization of $\mathrm{C}_{60}$-containing polymer composites and organic glasses (Ostroverkhova and Moerner, 2004)), based on our observation of significant PL quenching in ADT-TES-F/ $\mathrm{C}_{60}$ composite films compared to pristine ADT-TES-F films (Fig.5(b)) (Day et al., 2009a). Further addition of $\mathrm{C}_{60}$ lowered the measured photocurrent amplitude (e.g. by a factor of $\sim 2$ at $10 \%$ of $\mathrm{C}_{60}$, at $100 \mathrm{~V}$ ), most likely due to enhanced initial recombination, occurring at times below $\sim 100 \mathrm{ps}$, not resolved in our experiments. Addition of either TIPS-pentacene or ADT-TIPS-CN to ADT-TES-F lowered the amplitude of the transient photocurrent by a factor of $\sim 3-4$ at all applied voltages in the range studied. The most interesting effect of adding these molecules to the ADT-TES-F host was, however, the change in the initial photocurrent decay dynamics (Fig. 11). In particular,

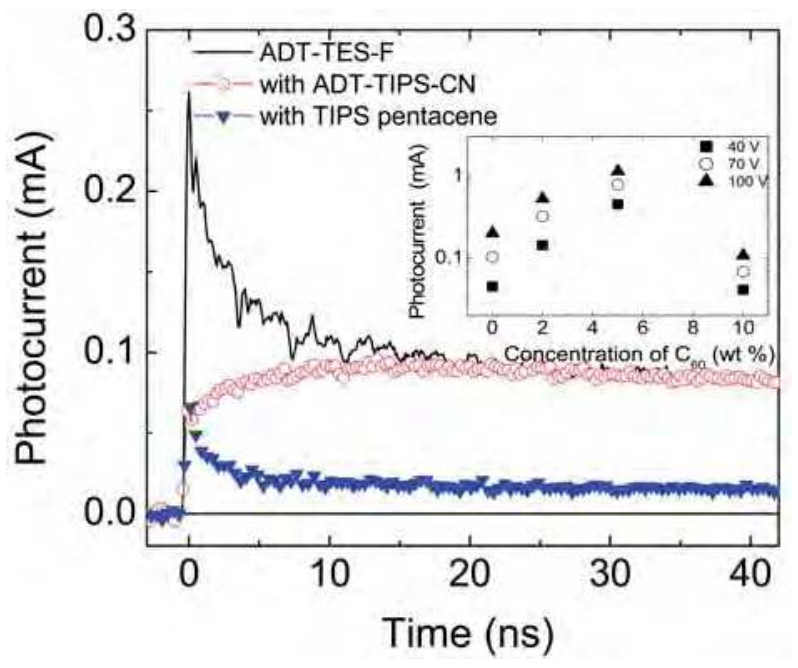

Fig. 11. Transient photocurrent obtained in pristine ADT-TES-F film and in ADT-TES-F/TIPSpentacene and ADT-TES-F/ADT-TIPS-CN composites at a fluence of $5 \mu \mathrm{J} / \mathrm{cm}^{2}$ at $100 \mathrm{~V}$ applied across $25 \mu \mathrm{m}$ gap. Inset shows amplitudes of the transient photocurrent as a function of concentration of $\mathrm{C}_{60}$ in ADT-TES-F/ $\mathrm{C}_{60}$ composites, measured at 40, 70, and $100 \mathrm{~V}$. Adapted from Day et al., 2009a, with permission. Copyright American Institute of Physics (2009). 
upon addition of TIPS-pentacene, the initial decay became faster, the effect similar to that achieved by the addition of $\mathrm{C}_{60}$. For example, only $\sim 32 \%$ of "initially" photogenerated carriers remained mobile at $5 \mathrm{~ns}$ after photoexcitation in the ADT-TES-F/TIPS-pentacene composite. We attribute this to fast hole transfer from ADT-TES-F to TIPS-pentacene followed by trapping at the TIPS-pentacene molecules (Day et al., 2009a). In contrast, addition of ADT-TIPS-CN to ADT-TES-F introduced a slow component into the photocurrent rise dynamics and completely removed the fast initial decay (Fig. 11). In particular, the fast rise of the photocurrent, limited by the time resolution of our setup, accounted only for $\sim 70 \%$ of all photogenerated carriers, whereas the other $30 \%$ were generated over $\sim 0.1-20 \mathrm{~ns}$. As a result, the peak of the photocurrent in the ADT-TES-F/ADTTIPS-CN composite was achieved at about $20 \mathrm{~ns}$ after photoexcitation, after which a slow decay, characterized by a power-law function with $\beta<0.1$, persisted to at least $1 \mathrm{~ms}$ after photoexcitation. Our observations of complete quenching of the PL of ADT-TES-F, while magnifying that of the ADT-TIPS-CN in the ADT-TES-F/ADT-TIPS-CN composite (Fig.5(b)) (Day et al., 2009a) suggest efficient energy transfer from ADT-TES-F to ADT-TIPS-CN. Therefore, it is possible that the slow component in the rise dynamics of the transient photocurrent in Fig. 11 is due to a multistep process that involves excitation of ADT-TES-F, followed by energy transfer to ADT-TIPS-CN, which then leads to energetically favorable hole transfer back to ADT-TES-F, while the electron remains trapped on the ADT-TIPS-CN molecules.

\subsection{Dark current}

Space-charge-limited currents (SCLCs) were observed in the ADT-TIPS-F, ADT-TES-F and TIPS-pentacene films on Au electrodes. Effective charge carrier mobilities $\left(\mu_{\text {eff }}\right)$ were calculated from the slope of the fits of the dark current as a function of applied voltage squared. In the case of planar electrode geometry used here, the current flows along a layer of unknown thickness, and there is no analytical solution for the relationship between SCLC (linear) density $\left(j=I_{d} / d\right.$, where $I_{d}$ is the dark current and $d$ is the channel width) and voltage $(\mathrm{V})$ in a film of finite thickness. In the approximation of the infinitely thin film, valid when the film thickness is much lower than the gap width (L) between the electrodes (which is the case here),

$$
\mathrm{j}=2 \mu_{\mathrm{eff}} \varepsilon \varepsilon_{0} \mathrm{~V}^{2} /\left(\pi \mathrm{L}^{2}\right),
$$

where $\varepsilon$ is a relative dielectric constant of the film and $\varepsilon_{0}$ is the dielectric permittivity of vacuum. SCLC effective mobilities $\left(\mu_{\mathrm{eff}}\right)$, which represent a lower bound of charge carrier mobilities in these films, showed sample-to-sample variation, especially significant in the case of ADT-TIPS-F and TIPS-pentacene (Table 3). On average, however, $\mu_{\text {eff }}$ in ADT-TES-F was at least a factor of $\sim 3$ higher than that in ADT-TIPS-F, and a factor of $\sim 7$ higher than in TIPS-pentacene films.

\subsection{Cw photocurrent}

Figure 12 shows cw photocurrent obtained at $532 \mathrm{~nm}$ excitation of ADT-TIPS-F, ADT-TES-F, and TIPS-pentacene films on interdigitated Au electrodes. In all samples, cw photocurrent measured at light intensity of $0.58 \mathrm{~mW} / \mathrm{cm}^{2}$ was higher than the dark current. Especially strong $\mathrm{cw}$ photoresponse was observed in best ADT-TES-F films, with photocurrents of over $200 \mu \mathrm{A}$ at the average electric field of $4 \times 10^{4} \mathrm{~V} / \mathrm{cm}$ at low light intensities (Fig. 12). This 


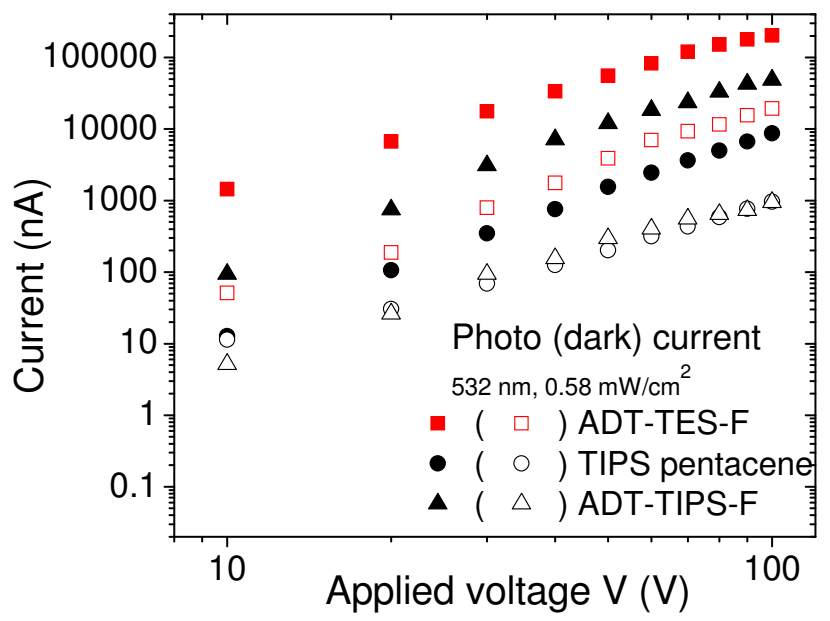

Fig. 12. Dark current and cw photocurrent at $532 \mathrm{~nm}$ excitation in ADT-TES-F, ADT-TIPS-F, and TIPS-pentacene films on interdigitated electrodes.

corresponds to linear photocurrent densities $\left(\mathrm{j}_{\mathrm{cw}}=\mathrm{I}_{\mathrm{cw}} / \mathrm{d}\right)$ of over $0.1 \mathrm{~mA} / \mathrm{cm}$, which, under assumption that photons absorbed throughout the entire thickness of a $1 \mu \mathrm{m}$ thick film may contribute to the photocurrent, yields (area) current densities $\left(\mathrm{J}_{\mathrm{cw}}\right)$ of over $1 \mathrm{~A} / \mathrm{cm}^{2}$. If photoconductivity $\left(\sigma_{\mathrm{ph}}\right)$ is calculated using $\sigma_{\mathrm{ph}}=\mathrm{J}_{\mathrm{cw}} / \mathrm{E}$, then values of $\sigma_{\mathrm{ph}} \sim 2.5 \times 10^{-5} \mathrm{~S} / \mathrm{cm}$ are obtained at $4 \times 10^{4} \mathrm{~V} / \mathrm{cm}$ at $0.58 \mathrm{~mW} / \mathrm{cm}^{2}$ in ADT-TES-F, which are considerably higher than those reported in conductive polymers such as PPV and in unsubstituted pentacene films or tetracene crystals under cw excitation (Platt et al., 2009b). As the temperature increased, the $\mathrm{cw}$ dc photocurrent increased, with the increase described by the function $\mathrm{I}_{\mathrm{cw}}$ $\sim \exp \left[-\Delta_{\mathrm{cw}} / \mathrm{k}_{\mathrm{B}} \mathrm{T}\right]$, with the activation energies $\Delta_{\mathrm{cw}}$ of $0.05-0.17 \mathrm{eV}$, depending on the sample and on the material (Day et al., 20081; Platt et al., 2009b). This is in contrast to the behavior observed on ps time-scales after photoexcitation in the same materials (Sections 3.3.1-3.3.2), which suggests a change in charge photogeneration and/or transport mechanisms as the time progresses.

Photoconductive gain $\mathrm{G}$ was calculated from the cw photocurrents, absorption coefficients, and light intensity as the ratio between the number of carriers flowing in the film and the number of absorbed photons. In the case of hole-transporting materials and hole-injecting electrodes (such as $\mathrm{Au}$ in the case of ADT-TIPS(TES)-F and TIPS-pentacene), bulk photoconductive gain

$$
\mathrm{G} \approx \eta_{0} \tau_{\mathrm{c}} / \mathrm{t}_{\mathrm{tr}}
$$

(where $\tau_{\mathrm{c}}$ is the carrier lifetime, and $t_{t r}$ is the time for the hole to transit through the film), and $G$ can be much larger than 1. Gain values $G$ that are much larger than unity were indeed observed in fluorinated ADT and TIPS-pentacene films, as summarized in Table 3. The highest photoconductive gains, up to 130 at $4 \times 10^{4} \mathrm{~V} / \mathrm{cm}$ at $0.58 \mathrm{~mW} / \mathrm{cm}^{2}$, were 
achieved in ADT-TES-F films, consistent with highest effective mobility $\mu_{\text {eff }}$ (shorter transit time $t_{\mathrm{tr}}$ ) and longest carrier lifetimes (as observed in SCLC and in the transient photocurrent measurements, respectively) in ADT-TES-F films, as compared to other materials under study. The values of G measured in ADT-TES-F films were similar to those in GaN photodetectors (Munoz et al.,1997) at similar light intensity levels and at least an order of magnitude higher than those in unsubstituted pentacene and in functionalized pentacene films (Gao \& Hegmann, 2008; Lehnherr et al., 2008).

\subsection{Influence of organic-metal interfaces on the current 3.6.1 Transient photocurrent}

Figure 13 (a) shows transient photocurrents, normalized at their peak values, measured in ADT-TIPS-F films on Au and Al electrodes. Interestingly, the faster decay component was more pronounced in films on $\mathrm{Al}$ electrodes, whereas the slower one was independent of the electrode material and could be described by a power-law function $\left(\mathrm{I}_{\mathrm{ph}} \sim \mathrm{t}^{-\beta}\right)$, as discussed above (Section 3.3.2). In samples with a $25-\mu \mathrm{m}$ gap at voltages above $\sim 30 \mathrm{~V}$, the difference in the peak transient amplitudes for films on $\mathrm{Au}$ and films on $\mathrm{Al}$ electrodes was comparable to that due to morphology-related sample-to-sample variation of approximately a factor of 2 (Fig. $13(b))$. The relation between the peak amplitude and applied voltage $\left(\mathrm{I}_{\mathrm{ph} \text {,max }} \sim \mathrm{V}^{\alpha}\right)$ was sample-dependent, with $\alpha=1.3-1.8$. Analysis of the behavior of many samples, however, yielded that on average, $\mathrm{a}$ in samples with $\mathrm{Al}$ electrodes was higher than in those with $\mathrm{Au}$

\begin{tabular}{|c|c|c|c|c|c|c|}
\hline Compound & $\begin{array}{c}\mu \eta, \mathrm{THz}^{\mathrm{a}} \\
\left(\mathrm{cm}^{2} /(\mathrm{Vs})\right.\end{array}$ & $\begin{array}{c}\mu \eta^{\mathrm{b}} \\
\left(\mathrm{cm}^{2} /(\mathrm{Vs})\right.\end{array}$ & $\begin{array}{c}\mu_{\text {eff }} \\
\left(\mathrm{cm}^{2} / \mathrm{Vs}\right)\end{array}$ & $\begin{array}{c}\mu_{\mathrm{FET}} \\
\left(\mathrm{cm}^{2} /(\mathrm{Vs})\right.\end{array}$ & $\eta_{0, \min ^{\mathrm{d}}}$ & $\mathrm{G}^{\mathrm{e}}$ \\
\hline ADT-TES-F & -- & $0.02-0.025$ & $0.033-0.092$ & $\begin{array}{c}1.7(\mathrm{film})^{\mathrm{f}} \\
6(\text { crystal) }\end{array}$ & $0.013-0.024$ & $70-130$ \\
\hline ADT-TIPS-F & -- & $0.018-0.025$ & $0.002-0.029$ & 0.1 (crystal) & $0.018-0.033$ & $16-30$ \\
\hline TIPS-pent. & $\begin{array}{c}0.02-0.06 \\
(\text { film) } \\
0.15-0.2 \\
\text { (crystal) }\end{array}$ & $0.01-0.022$ & $0.002-0.007$ & $1.5($ film) & $0.0003-0.001$ & $9-28$ \\
\hline
\end{tabular}

Table 3. (Photo)conductive properties of TIPS-pentacene and ADT thin films.

a Calculated from the peak THz transmission using Eq.(2) (Section 3.3.1 and Ostroverkhova et al., 2005b)

$\mathrm{b}$ Calculated from the peak of the transient photocurrent measured at $400 \mathrm{~nm}$ excitation in thin-film device structures using DSO (Section 3.3.2 and Day et al., 2008)

c Calculated from SCLC currents in thin-film devices (Section 3.4 and Platt et al., 2009b)

$\mathrm{d}$ Calculated from cw photocurrents in thin-film devices on $\mathrm{Al}$ electrodes (Section 3.5 and Platt et al., 2009b)

e Calculated from cw photocurrents in thin-film devices on Au electrodes (Section 3.4 and Platt et al., 2009b)

f Calculated from TFT characteristics of ADT-TES-F spin-coated onto Au electrodes treated with PFBT (Park et al., 2008)

g Calculated from FET characteristics of ADT-TES-F single crystal (Jurchescu et al., 2008)

$\mathrm{h}$ Calculated from FET characteristics of ADT-TIPS-F single crystal (Subramanian et al., 2008)

i Calculated from TFT characteristics of spin-coated TIPS-pentacene film (Park et al., 2007) 
electrodes (e.g. $\alpha=1.76 \pm 0.06$ and $1.33 \pm 0.01$ in ADT-TIPS-F films on Al and Au electrodes, respectively, in Fig. 13 (b)). Similar dependencies were observed for all electrode geometries, regardless of film-side or glass substrate-side illumination. Since measurements of the photocurrent were performed in the presence of applied electric field, the photoresponse was necessarily measured after dark current flow had been established in the film, or, in other words, after the sample had been pre-conditioned by the dark current. In samples with $\mathrm{Au}$ and $\mathrm{Al}$ electrodes, very different charge injection conditions obtain and, therefore, different pre-conditioning of the samples is realized. In particular, samples with $\mathrm{Au}$ electrodes, in which SCLC regime is established (Section 3.4), are expected to have a high density of filled hole traps. In contrast, samples with $\mathrm{Al}$ electrodes are expected to be in a close-to-pristine condition (empty traps) at low voltages, and have partially filled traps at higher voltages, after hole injection via, for example, Fowler-Nordheim tunneling is enabled (Day et al., 2009b). If fast initial decay is related to bulk material properties and is due to fast trapping of photogenerated holes, then in films on Au electrodes, the density of empty traps is reduced, which in turn reduces initial trapping of the photogenerated holes. In contrast, most traps in films on $\mathrm{Al}$ electrodes are empty and are readily filled by photogenerated holes, thus leading to a fast initial decay of the photocurrent. This is also consistent with previously observed slowing down of the initial decay of the transient photocurrent upon increasing applied electric field in ADT-TIPS-F films on Al electrodes (Day et al., 2008).

(a)

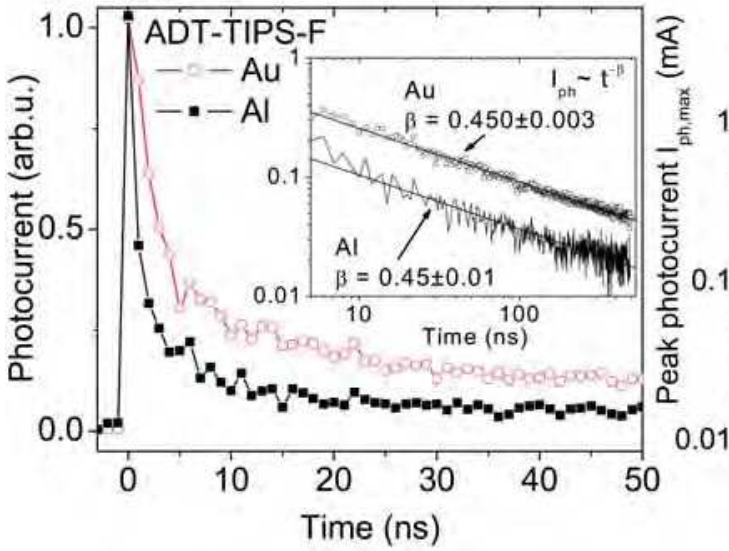

(b)

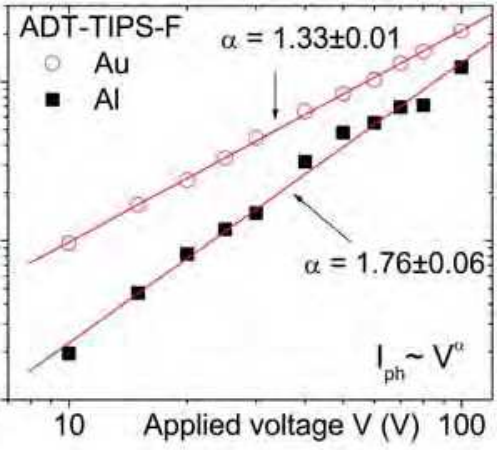

Fig. 13. (a) Transient photocurrents, normalized at their peak values, obtained in ADT-TIPSF films on $\mathrm{Au}$ and $\mathrm{Al}$ electrodes under the same experimental conditions. Inset: long timescale dynamics of the same transient photocurrents. Power-law fits $\left(I_{\mathrm{ph}} \sim t^{-\beta}\right)$ are also shown. (b) Transient photoresponse peak amplitude $\left(I_{\mathrm{ph} ; \max }\right)$ as a function of applied voltage obtained in ADT-TIPS-F samples with Al or Au interdigitated electrodes. Reprinted from Day et al., 2009b, with permission. Copyright American Institute of Physics (2009).

Scanning microscopy. The peak amplitudes of the transient photocurrent measured at different positions of the localized pulsed photoexcitation of ADT-TES-F and ADT-TIPS-F films on $\mathrm{Au}$ and $\mathrm{Al}$ electrodes are shown in Fig. 14 (a). No signal was detected at any position in the absence of applied voltage. Change in the transient photocurrent amplitude upon scanning of the pulsed focused beam across a planar device has been previously 
related to internal electric field distribution in InP planar devices (Day et al., 2009b). In ADT films, although the exact distribution of transient photocurrent amplitudes in the gap somewhat depended on the measurement protocol (such as direction of the scan and waiting time between the data points), these distributions in samples with $\mathrm{Au}$ and $\mathrm{Al}$ electrodes were similar within the experimental error and, with our spatial resolution, relatively uniform across the gap. In particular, the amplitudes of transient photocurrents obtained upon excitation of near-electrode regions and of a mid-gap region were within a factor of 2 of each other. Transient photocurrent decay dynamics under localized excitation in samples with $\mathrm{Au}$ and $\mathrm{Al}$ electrodes were similar to those in Fig. 13 (a) obtained under uniform illumination, with the initial decay faster in samples with Al electrodes. No dependence of the decay dynamics of the transient photocurrent on the beam position in the gap was observed.

(a)
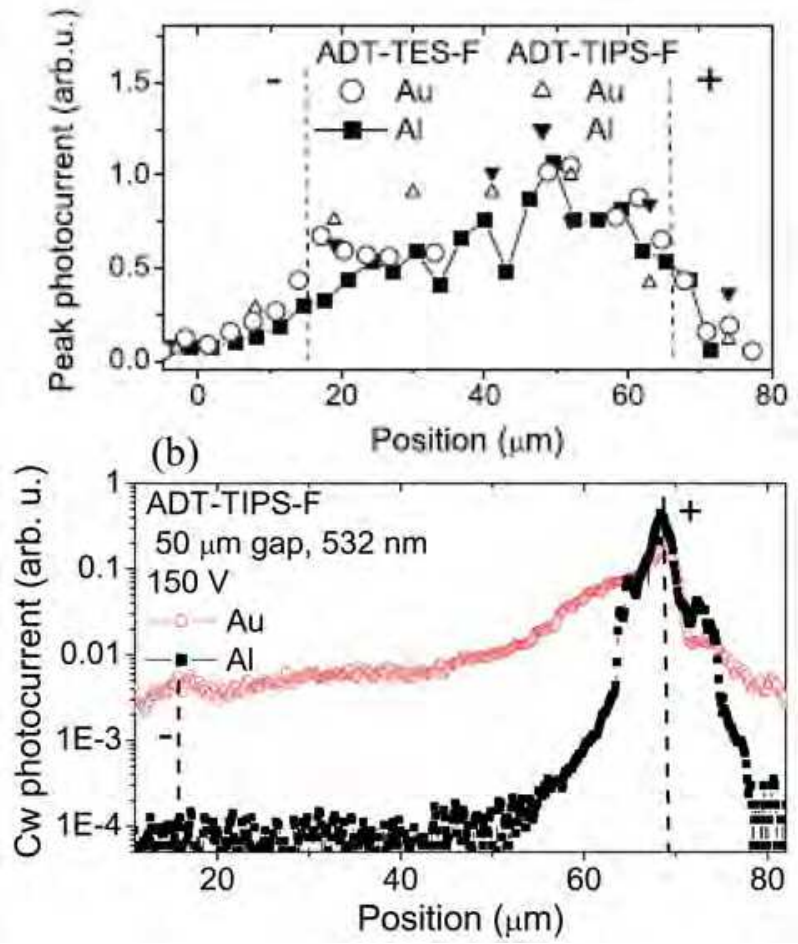

Fig. 14. (a) Peak amplitudes of the transient photocurrent, normalized at their maximal values in the gap, at different positions of the localized beam spot obtained in ADT-TES-F and ADT-TIPS-F films on Au and Al electrodes, in coplanar electrode geometry with $50-\mu \mathrm{m}$ gap at $100 \mathrm{~V}$ and $150 \mathrm{~V}$, respectively. (b) $\mathrm{Cw}$ photoresponse as a function of the beam position obtained in an ADT-TIPS-F film in coplanar electrode geometry with $50-\mu \mathrm{m}$ gap with $\mathrm{Al}$ and $\mathrm{Au}$ electrodes at $150 \mathrm{~V}$. Dashed lines correspond to the geometrical edges of the electrodes. Adapted from Day et al., 2009b, with permission. Copyright American Institute of Physics (2009). 


\subsubsection{Dark current and cw photocurrent}

Unlike the amplitudes of transient photocurrent, the cW photocurrents $\left(\mathrm{I}_{\mathrm{cW}}\right)$ and dark currents $\left(\mathrm{I}_{\mathrm{d}}\right)$ for films on $\mathrm{Au}$ and on $\mathrm{Al}$ electrodes differed by more than an order of magnitude over a wide range of applied voltages, with much higher currents measured in samples with Au electrodes (Fig. 15). Voltage dependencies of the dark current and of the $\mathrm{cw}$ photocurrent observed in samples with $\mathrm{Au}$ and $\mathrm{Al}$ electrodes were drastically different, indicative of different mechanisms responsible for observed currents, depending on the electrode material. In samples with Au electrodes, the relationship between dark current and voltage $\left(\mathrm{I}_{\mathrm{d}} \sim \mathrm{V}^{\alpha_{\mathrm{d}}}\right)$ was close to linear at very low voltages and close to quadratic at higher voltages (e.g. $\alpha_{d}=1.09 \pm 0.03$ at voltages below $2 \mathrm{~V}$ and $1.830 \pm 0.006$ from 2 to $300 \mathrm{~V}$ in an ADT-TES-F film in Fig. 15(a)). In contrast, in samples with Al electrodes, dark current was weakly voltage-dependent at lower voltages, followed by a steep increase at higher voltages. Despite this sharp increase, even at the highest applied voltage of $300 \mathrm{~V}$ used in our experiments, dark current in samples with $\mathrm{Al}$ electrodes was much lower than that in samples with $\mathrm{Au}$ electrodes at the same voltage. In films on either $\mathrm{Au}$ or $\mathrm{Al}$ electrodes, the $\mathrm{cw}$ photocurrent was higher than the dark current in the entire range of light intensities used. Regardless of the material, in samples with Au electrodes, $\alpha_{\mathrm{cw}}$ obtained from the fit of voltage dependence of the photocurrent $\left(\mathrm{I}_{\mathrm{cw}} \sim \mathrm{V}^{\alpha}{ }_{\mathrm{cw}}\right)$ ranged between 1.5 and 2.2, depending on the sample. In any given sample, however, a single value of $\alpha_{c w}$ was sufficient to describe voltage dependence of the $\mathrm{cw}$ photocurrent over a large voltage range (e.g. $\alpha_{\mathrm{cw}}=1.62 \pm 0.02$ from 5 to $300 \mathrm{~V}$ in an ADT-TES-F film in Fig. 15(b)). In samples with Al electrodes, however, $\alpha_{\mathrm{cw}}$ was $\sim 1.3-3$ at lower voltages (e.g. $\alpha_{\mathrm{cw}}=1.32 \pm 0.05$ in Fig. $15(\mathrm{~b})$ ), depending on the sample, followed by steep transition described by $\alpha_{c w} \sim 3.3-5$ (e.g. $\alpha_{c w}=4.6 \pm 0.2$ in Fig. $\left.15(b)\right)$ at higher voltages. Similar trends were observed in ADT-TIPS-F films. (Day et al., 2009b).

(a)

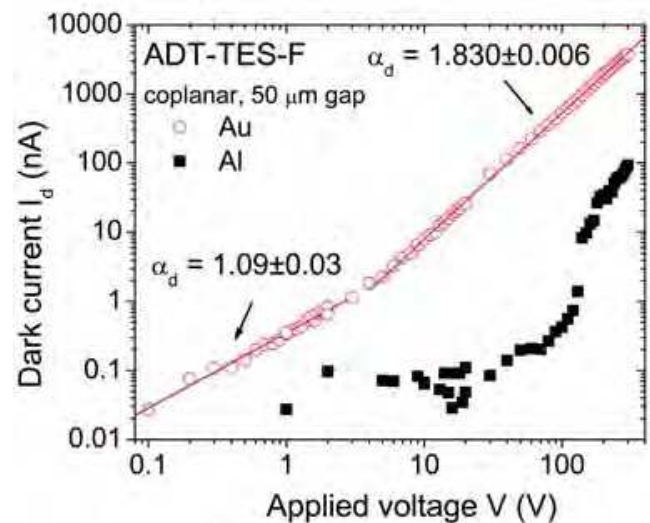

(b)

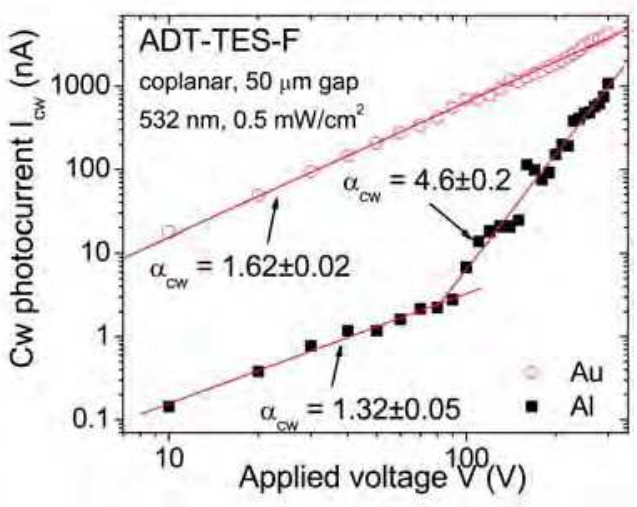

Fig. 15. (a) Dark current $\left(I_{\mathrm{d}}\right)$ and (b) cw photocurrent $\left(I_{\mathrm{cw}}\right)$ obtained in ADT-TES-F films with $\mathrm{Al}$ and Au contacts in coplanar geometry with 50- $\mu \mathrm{m}$ gap. Power-law fits $I_{\mathrm{d}} \sim V^{\alpha_{\mathrm{d}}}$ and $I_{\mathrm{cw}} \sim$ $V^{\alpha_{\mathrm{cw}}}$ in (a) and (b), respectively, are also shown.

The photoresponse under cw illumination is strongly affected by the conditions imposed by the electrode material (Fig. 15(b)) because the steady-state photocurrent $\left(\mathrm{I}_{\mathrm{cw}}\right)$ is proportional to the photoconductive gain $(\mathrm{G})$, which is much larger than 1 in our samples on $\mathrm{Au}$ 
electrodes (Table 3) due to long carrier lifetimes (Day et al., 2009b). In samples with noninjecting electrodes, such as ADT films on Al electrodes at low voltages, the maximal achievable gain cannot exceed the initial photogeneration efficiency $\eta_{0}\left(\eta_{0}<1\right)$ and therefore, the $\mathrm{cw}$ photocurrent $\mathrm{I}_{\mathrm{cw}}$ is much lower. This is in contrast to the transient photocurrent, the amplitude of which does not significantly depend on the carrier lifetime, thus leading to comparable transient photoresponse of films on Au and Al electrodes.

In samples with blocking electrodes (such as Al in the case of ADT-TIPS(TES)-F and TIPS pentacene), $G$ cannot exceed $\eta_{0}$. Thus, the photoconductive gain calculated from $\mathrm{cw}$ photocurrents measured in samples with $\mathrm{Al}$ electrodes at low voltages represents a lower limit of the photogeneration efficiency $\eta_{0, \text { min. }}$. In ADT-TES-F and ADT-TIPS-F films, comparable values of $\eta_{0, \min } \approx 0.01-0.03$ were obtained at $1.2 \times 10^{4} \mathrm{~V} / \mathrm{cm}$ (Table 3). Interestingly, these values are similar to those estimated from transient photocurrents measured in the same films. In constrast, in TIPS-pentacene films, the $\eta_{0, \text { min }}$ values were considerably lower than those calculated from the amplitudes of the transient photocurrents (Table 3). This is most likely due to extensive deep trapping in TIPS pentacene, which reduces carrier lifetime, leading to an underestimation of the initial photogeneration efficiency $\eta_{0}$ from $G$.

Scanning microscopy. Figure 14 (b) shows the $\mathrm{cw}$ photoresponse measured under localized excitation of ADT-TIPS-F films on Al and Au electrodes, respectively, as a function of the beam position in the gap. Samples with either $\mathrm{Al}$ or Au electrodes showed a marked peak in photoresponse at the excitation near the positively biased electrode, which became more pronounced as the voltage increases. This effect was independent of the direction of the scan, was observed at all light powers ranging between 0.03 and $4 \mu \mathrm{W}$ used in our experiments, and was attributed to a dominant photogenerated hole transport, accompanied by strong electron trapping (Day et al., 2009b). As seen from Fig. 14(b), at $150 \mathrm{~V}$, in the case of $\mathrm{Al}$ electrodes, photoresponse was mostly limited to the several micron-wide region. Under the same conditions, photoresponse in the sample with Au electrodes was more uniform within the gap (Fig. 14(b)), yielding measurable photocurrents upon excitation of the mid-gap region, as well as of the region close to the negatively biased electrode. Interestingly, if the waiting period between the successive scans was short compared to the time needed to thermally empty the filled charge traps, the photocurrent distribution in the gap became more and more uniform with each scan, which could be due to increased carrier lifetime under trap-filled conditions. No photoresponse was observed in the absence of an applied voltage (Day et al., 2009b). Such pronounced differences in cw photoresponse behavior of samples on $\mathrm{Au}$ and $\mathrm{Al}$ electrodes are in agreement with results obtained under uniform illumination in the same devices (Fig. 15).

\section{Discussion}

One of the challenging aspects of dealing with organic semiconductors, especially thin films, is that intrinsic properties are often masked by impurities, grain boundaries, and defects. As a result, it is often difficult to reconcile results of different experiments performed on the same material. One of our motivations for the studies described above was to develop a picture of charge photogeneration and transport in acenes that would be consistent with results of a variety of experiments that probe carrier and exciton dynamics from sub-ps to many seconds after photoexcitation. 
First of all, it seems clear that charge carriers in acenes can be created via photoexcitation on time-scales of ps and faster, without either thermal or electric-field assistance. However, in keeping with $\mu \eta$ values extracted from the transient photoconductivity experiments (Sections 3.3.1 and 3.3.2) and assuming intrinsic mobilities at room temperature in most of our materials to be below $10 \mathrm{~cm}^{2} /(\mathrm{Vs})$ (Park et al., 2007; Jurchescu et al., 2008; Park et al., 2008, Subramanian et al., 2008), the efficiency of this process is rather low, with initial photogeneration efficiencies $\eta_{0}$ of $<5 \%$. This is also consistent with the estimates of the $\eta_{0}$ from cw photocurrent measurements (Table 3). The other $>95 \%$ of the primary photoexcitation results in exciton formation. The origin of the photogenerated carriers observed in transient photocurrent experiments on time scales from sub-ps to ns after photoexcitation does not appear to be excitonic (i.e. due to exciton dissociation) due to the following observations: (i) decrease of $\mu \eta$ with the temperature, as opposed to an increase expected from the Onsager model of exciton dissociation; (ii) weak, if any, wavelength dependence of the photogeneration efficiency above the absorption edge; (iii) similar $\mu \eta$ values for TIPS-pentacene and functionalized ADTs, despite very different exciton behavior in films (high PL QYs in ADTs and ultra-low ones in TIPS-pentacene); (iv) different charge carrier and exciton dynamics (long power-law and fast bi-exponential, respectively), assessed using time-resolved photocurrent and PL, respectively; and (v) no apparent electric field-induced exciton dissociation up to electric fields that are more than an order of magnitude higher than those used in photocurrent measurements. Photogeneration efficiency can be improved by introducing a strong acceptor such as $\mathrm{C}_{60}$ as a guest molecule in the acene host due to fast photoinduced electron transfer from the host molecule to $\mathrm{C}_{60}$, which releases a free hole into the host. Other dopants can affect the photoexcited charge carrier dynamics as well by initiating competition between energy and charge transfer.

It is also interesting to note that $\mu \eta$ values obtained from direct measurements of the transient photocurrent (Section 3.3.2) in TIPS-pentacene solution-deposited films at electric fields of $\sim 10^{4} \mathrm{~V} / \mathrm{cm}$ are only about a factor of 2-3 lower than those measured at earlier times in the optical pump-THz probe experiments (Section 3.3.1) in similar films at zero static electric field (Table 3). Since at low electric fields neither mobility nor photogeneration efficiency are expected to strongly depend on the electric field, this difference would be mostly due to a carrier loss that occurs between $\sim 400 \mathrm{fs}$ and $\sim 30$ ps after photoexcitation, which correspond to the time resolution of these two experiments.

Regarding charge transport mechanism, the following picture seems to apply. At ps timescales after photoexcitation, charge transport is band-like, which is characterized by mobility increasing as the temperature decreases and by mobility anisotropy in crystals. As the time progresses, dispersive transport takes over and persists over many orders of magnitude in time. Interestingly, in functionalized acenes, it appears that non-activated carrier tunneling, rather than hopping, occurs at least up to ns after photoexcitation, since no significant temperature dependence of the transient photoconductivity power-law decay dynamics was observed in the wide temperature range. The fact that this behavior is seen in both single crystals and thin films suggests that it is an intrinsic property of these materials, possibly related to molecular $\pi$-stacking. In the equilibrium, the transport is thermally activated, consistent with hopping mechanism. An interesting feature of these materials is persistent photoconductivity due to long carrier lifetimes, which lead to high photoconductive gains. Equilibrium transport is characterized by the effective mobility $\mu_{\text {eff }}=\mu \theta$, where $\theta$ is the ratio between free and trapped charge carriers $(\theta \leq 1)$. In our films, $\mu_{\mathrm{eff}}$ extracted from SCLC 
measurements (Section 3.4) is lower than the intrinsic mobility $\mu$, since most likely the trapfree limit has not been achieved in our samples (Table 3). Indeed, although $\mu_{\text {eff }}$ values of $0.033-0.092 \mathrm{~cm}^{2} /$ Vs obtained in ADT-TES-F films were similar to those obtained in ADTTES-F TFTs with channel lengths similar to ours (i.e. $25 \mu \mathrm{m}$ ), mobilities of over $1.5 \mathrm{~cm}^{2} / \mathrm{Vs}$ and $6 \mathrm{~cm}^{2} /$ Vs have been achieved in short-channel ADT-TES-F films spin-coated on the Au electrodes treated with pentafluorobenzenethiol (PFTB) (Park et al., 2008) and in ADT-TES-F single crystals (Jurchescu et al., 2008), respectively. Therefore, $\theta$ is below $\sim 0.015$ in our ADTTES-F films. It is lower yet in TIPS-pentacene films, since $\mu_{\text {eff }}$ values of $0.002-0.007 \mathrm{~cm} 2 / \mathrm{Vs}$ obtained in our SCLC measurements are much lower than $1.2 \mathrm{~cm}^{2} /$ Vs observed in TIPSpentacene TFTs, indicative of high trap density in our TIPS-pentacene films.

Also, under equilibrium, there is a possible interesting connection between the PL and cw photocurrent. In particular, temperature dependencies of the PL QYs and cw photoconductivity, characterized by $\Delta_{\mathrm{PL}}$ and $\Delta_{\mathrm{cw}}$, respectively (Sections 3.1 and 3.4), seem to be close to each other in the same samples (Platt et al., 2009b). It has been shown theoretically that in materials with $\pi$-stacking, such as functionalized pentacene and ADT derivatives, thermal fluctuations lead to dramatic changes in intermolecular coupling (Troisi and Orlandi, 2006), which could be important for both PL and photoconductivity in films. In particular, it appears that thermally activated nuclear motions can both enhance nonradiative recombination, leading to a decrease in the PL, and facilitate charge carrier detrapping from shallow traps and transport of localized carriers, which would improve cw photoconductivity. This does not seem to affect transient photocurrents measured at ps time-scales after photoexcitation.

Finally, the electrode material plays a significant role in the dark current and cw photoresponse, since both rely on efficient carrier injection from the electrode. In contrast, transient photocurrents are only weakly affected by the choice of the electrodes.

\section{Conclusions and outlook}

Over the past decade, tremendous progress has been made towards understanding of physical mechanisms that determine optical and electronic properties of organic semiconductors, in development of novel experimental methods that probe intrinsic properties of materials, in organic synthesis and purification of materials, and in fabrication and characterization of various devices. Novel solution-processable high-purity materials with tunable properties are now available, and a variety of methods of high-quality crystal growth and thin-film deposition have been developed. A number of devices that utilize organic thin-film transistors and light-emitting diodes are already on the market, and performance of many more devices, such as photovoltaic cells and various photorefractive devices, keeps steadily improving and has already almost reached the point of commercial feasibility. Possibility to fine-tune optical and electronic properties by creating solutiondeposited organic semiconductor composite films offers an extraordinary flexibility to tailor the materials for the specific application demands. Another exciting possibility is to utilize properties of individual highly photoluminescent molecules (such as ADT-TES-F) as nanoprobes of conduction, nanoswitches, and nano optoelectronic devices.

Despite considerable progress, many issues require further attention. In particular, the exact nature of charge photogeneration and transport in small-molecular-weight organic semiconductors is still not well understood, and new experimental methods reveal 
additional layers of complexity of their behavior. Also, more solution-processable materials with improved charge-transfer properties are in demand. Moreover, optical and electronic properties strongly depend on the structure and morphology of organic films and, therefore, fabrication methods that yield consistent films, and over a large substrate area, are needed. Finally, influence of ambient conditions, including temperature, humidity, the presence of oxygen and other gases, etc. on device performance is strong and, ideally, has to be minimized by creative design of device structures. Nevertheless, the field of organic photonics and electronics is developing fast and will undoubtedly produce new and exciting results in the near future.

\section{Acknowledgements}

We are indebted to Profs. F. A. Hegmann and D. G. Cooke for the facilities guidance, and contribution to THz spectroscopy work. We thank Profs. J. E. Anthony, V. Podzorov, M. E. Gershenson, O. D. Jurchescu, and T. T. M. Palstra for organic materials used in our work. Technical assistance of Prof. Y.S. Lee, Dr. J. Danielsen, J. Tomaino, and R. Presley is acknowledged. Also thanks to Dr. J. Day for carrying out initial measurements of photoconductive properties of materials in our group. This work was supported by the Killam Trust, Office of Naval Research (grant \#N00014-07-1-0457 via ONAMI Nanometrology and Nanoelectronics Initiative) and National Science Foundation via CAREER program (DMR-0748671).

\section{References}

Agostinelli, T., Caironi, M., Natali, D., Sampietro, M., Biagioni, P., Finazzi, M. and Duo, L. (2007). "Space charge effects on the active region of a planar organic photodetector." Journal of Applied Physics 101, 114504.

Ahn, Y. H., Tsen, A. W., Kim, B., Park, Y. W. and Park, J. (2007). "Photocurrent imaging of pn junctions in ambipolar carbon nanotube transistors." Nano Letters 7(11), 3320-3323.

Anthony, J. E., Brooks, J. S., Eaton, D. L. and Parkin, S. R. (2001). "Functionalized pentacene: Improved electronic properties from control of solid-state order." Journal of the American Chemical Society 123(38), 9482-9483.

Anthony, J. E. (2006). "Functionalized acenes and heteroacenes for organic electronics." Chemical Reviews 106, 5028-5048.

Brutting, W., Ed. (2005). Physics of Organic Semiconductors. Weinheim, Wiley-VCH.

Cheng, Y. C. and Silbey, R. J. (2008). "A unified theory for charge-carrier transport in organic crystals." Journal of Chemical Physics 128, 114713.

Coropceanu, V., Cornil, J., da Silva, D., Olivier, Y., Silbey, R. J. and Bredas, J. L. (2007). "Charge transport in organic semiconductors." Chemical Reviews 107, 926-952.

Day, J., Subramanian, S., Anthony, J. E., Lu, Z., Twieg, R. J. and Ostroverkhova, O. (2008). "Photoconductivity in organic thin films: from picoseconds to seconds after excitation." Journal of Applied Physics 103, 123715.

aDay, J., Platt, A. D., Ostroverkhova, O., Subramanian, S. and Anthony, J. E. (2009). "Organic semiconductor composites: influence of additives on the transient photocurrent." Applied Physics Letters 94(1), 013306.

bDay, J., Platt, A. D., Subramanian, S., Anthony, J. E. and Ostroverkhova, O. (2009). "Influence of organic semiconductor-metal interfaces on the photoresponse of functionalized anthradithiophene thin films." Journal of Applied Physics 105, 103703. 
de Boer, R. W. I., Jochemsen, M., Klapwijk, T. M., Morpurgo, A. F., Niemax, J., Tripathi, A. K. and Pflaum, J. (2004). "Space charge limited transport and time of flight measurements in tetracene single crystals:a comparative study." Journal of Applied Physics 95(3), 1196-1202.

Etemad, S., Mitani, T., Ozaki, M., Chung, T. C., Heeger, A. J. and MacDiarmid, A. G. (1981). "Photoconductivity in polyacetylene." Solid State Communications 40, 75-79.

Forrest, S. R. (2004). "The path to ubiquitous and low-cost organic electronic appliances on plastic." Nature 428, 911-918.

Gao, J. and Hegmann, F. A. (2008). "Bulk photoconductive gain in pentacene thin films." Applied Physics Letters 93(22), 223306.

Gu, Y., Romankiewicz, J. P., David, J. K., Lensch, J. L. and Lauhon, L. J. (2006). "Quantitative measurement of the electron and hole mobility-lifetime products in semiconductor nanowires." Nano Letters 6(5), 948-952.

Hegmann, F. A., Ostroverkhova, O. and Cooke, D. G. (2005). Probing Organic Semiconductors with Terahertz Pulses. Photophysics of molecular materials. G. Lanzani. Weinheim, Wiley-VCH.

Hegmann, F. A., Tykwinski, R. R., Lui, K. P. H., Bullock, J. E. and Anthony, J. E. (2002). "Picosecond transient photoconductivity in functionalized pentacene molecular crystals probed by terahertz pulse spectroscopy." Physical Review Letters 89(22), 227403.

Jurchescu, O. D., Baas, J. and Palstra, T. T. M. (2004). "Effect of impurities on the mobility of single crystal pentacene." Applied Physics Letters 84, 3061-3063.

Jurchescu, O. D., Subramanian, S., Kline, R. J., Hudson, S. D., Anthony, J. E., Jackson, T. N. and Gundlach, D. J. (2008). "Organic single-crystal field-effect transistors of a soluble anthradithiophene." Chemistry of Materials 20(21), 6733-6737.

Karl, N. (2001). Charge-carrier mobility in organic crystals. Organic Electronic Materials. R. Farchioni and Grosso, G. New York, Springer, 449.

Karl, N. (2003). "Charge carrier transport in organic semiconductors." Synthetic Metals 133, 649-657.

Knipp, D., Street, R. A., Volkel, A. and Ho, J. (2003). "Pentacene thin film transistors on inorganic dielectrics: Morphology, structural properties, and electronic transport." Journal of Applied Physics 93(1), 347-355.

Koeberg, M., Hendry, E., Schins, J. M., Laarhoven, H. A. v., Flipse, C. F. J., Reimann, K., Woerner, M., Elsaesser, T. and Bonn, M. (2007). "Simultaneous ultrafast probing of intramolecular vibrations and photoinduced charge carriers in rubrene using broadband time-domain THz spectroscopy." Physical Review B 75, 195216.

Laarhoven, H. A. v., Flipse, C. F. J., Koeberg, M., Bonn, M., Hendry, E., Orlandi, G., Jurchescu, O. D., Palstra, T. T. M. and Troisi, A. (2008). "On the mechanism of charge transport in pentacene." Journal of Chemical Physics 129, 044704.

Lang, D. V., Chi, X., Siegrist, T., Sergent, A. M. and Ramirez, A. P. (2004). "Amorphouslike density of gap states in single-crystal pentacene." Physical Review Letters 93(8), 086802.

Lehnherr, D., Gao, J., Hegmann, F. A. and Tykwinski, R. R. (2008). "Synthesis and electronic properties of conjugated pentacene dimers." Organic Letters 10(21), 4779-4782.

Lord, S. J., Lu, Z., Wang, H., Willets, K. A., Schuck, P. J., Lee, H. D., Nishimura, S. Y., Twieg, R. J. and Moerner, W. E. (2007). "Photophysical properties of acene DCDHF fluorophores: long-wavelength single-molecule emitters designed for cellular imaging." Journal of Physical Chemistry A 111, 8934-8941. 
Lui, K. P. H. and Hegmann, F. A. (2001). "Ultrafast carrier relaxation in radiation-damaged silicon-on-sapphire studied by optical pump-terahertz-probe experiments." Applied Physics Letters 78, 3478-3480.

Marciniak, H., Pugliesi, I., Nickel, B. and Lochbrunner, S. (2009). "Ultrafast singlet and triplet dynamics in microcrystalline pentacene films." Physical Review B 79, 235318.

Moses, D. (1989). "Charge quantum yield and transient transport properties of anthracene and amorphous selenium." Solid State Communications 69(7), 721-725.

Moses, D., Dogariu, A. and Heeger, A. J. (2000). "Ultrafast detection of charged photocarriers in conjugated polymers." Physical Review B 61(14), 9373-9379.

Moses, D., Sinclair, M. and Heeger, A. J. (1987). "Carrier Photogeneration and Mobility in Polydiacetylene - Fast Transient Photoconductivity." Physical Review Letters 58(25), 2710-2713.

Moses, D., Soci, C., Chi, X. and Ramirez, A. P. (2006). "Mechanism of carrier photogeneration and carrier transport in molecular crystal tetracene." Physical Review Letters 97, 067401.

Munoz, E., Monroy, E., Garrido, J. A., Izpura, I., Sanchez, F. J., Sanchez-Garcia, M. A., Calleja, E., Beaumont, B. and Gibart, P. (1997). "Photoconductor gain mechanisms in GaN ultraviolet detectors." Applied Physics Letters 71(7), 870-872.

Murphy, A. R. and Frechet, J. M. J. (2007). "Organic semiconducting oligomers for use in thin film transistors." Chemical Reviews 107, 1066-1096.

Najafov, H., Lyu, B. and Biaggio, I. (2008). "Investigating the origin of the high photoconductity of rubrene single crystals." Physical Review B 77, 125202.

Nelson, S. F., Lin, Y. Y., Gundlach, D. J. and Jackson, T. N. (1998). "Temperatureindependent transport in high-mobility pentacene transistors." Applied Physics Letters 72(15), 1854-1856.

Newman, C. R., Frisbie, C. D., da Silva Filho, D., Bredas, J. L., Ewbank, P. C. and Mann, K. R. (2004). "Introduction to organic thin film transistors and design of n-channel organic semiconductors." Chemistry of Materials 16, 4436-4451.

Ostroverkhova, O. and Moerner, W. E. (2004). "Organic photorefractives: mechanisms, materials and applications." Chemical Reviews 104(7), 3267-3314.

astroverkhova, O., Cooke, D. G., Shcherbyna, S., Egerton, R. F., Hegmann, F. A., Tykwinski, R. R. and Anthony, J. E. (2005). "Band-like transport in pentacene and functionalized pentacene thin films revealed by transient photoconductivity." Physical Review B 71, 035204.

bOstroverkhova, O., Shcherbyna, S., Cooke, D. G., Egerton, R. F., Hegmann, F. A., Tykwinski, R. R., Parkin, S. R. and Anthony, J. E. (2005). "Optical and transient photoconductive properties of pentacene and functionalized pentacene thin films: Dependence on film morphology." Journal of Applied Physics 98, 033701.

a Ostroverkhova, O., Cooke, D. G., Hegmann, F. A., Anthony, J. E., Podzorov, V., Gershenson, M. E., Jurchescu, O. D. and Palstra, T. T. M. (2006). "Ultrafast carrier dynamics in pentacene, functionalized pentacene, tetracene and rubrene single crystals." Applied Physics Letters 88, 162101.

bOstroverkhova, O., Cooke, D. G., Hegmann, F. A., Tykwinski, R. R., Parkin, S. R. and Anthony, J. E. (2006). "Anisotropy of transient photoconductivity in functionalized pentacene single crystals." Applied Physics Letters 89, 192113.

Park, J., Ahn, Y. H. and Ruiz-Vargas, C. (2009). "Imaging of photocurrent generation and collection in single-layer graphene." Nano Letters 9(5), 1742-1746. 
Park, S. K., Jackson, T. N., Anthony, J. E. and Mourey, D. A. (2007). "High mobility solution processed 6,13-bis(triisopropyl-silylethynyl)pentacene organic thin film transistors." Applied Physics Letters 91, 063514.

Park, S. K., Mourey, D. A., Subramanian, S., Anthony, J. E. and Jackson, T. N. (2008). "Highmobility spin-cast organic thin film transistors." Applied Physics Letters 93, 043301.

Peumans, P., Yakimov, A. and Forrest, S. R. (2003). "Small molecular weight organic thinfilm photodetectors and solar cells." Journal of Applied Physics 93(7), 3693-3723.

aPlatt, A. D., Day, J., Shepherd, W. E. B. and Ostroverkhova, O. (2009). Photophysical and photoconductive properties of novel organic semiconductors, in Organic Thin Films for Photonic Applications (Ed.S. H. Foulger), Washington, D.C., ACS.

bPlatt, A. D., Day, J., Subramanian, S., Anthony, J. E. and Ostroverkhova, O. (2009). "Optical, fluorescent, and photoconductive properties of high-performance functionalized pentacene and anthradithiophene derivatives." Journal of Physical Chemistry $C$ in press.

cPlatt, A. D., Shepherd, W. E. B., Anthony, J. E. and Ostroverkhova, O. (2009). "Photophysical and photoconductive properties of organic semiconductor composites." Proc. of SPIE 7413, 7413-OS.

Podzorov, V., Pudalov, V. M. and Gershenson, M. E. (2003). "Field-effect transistors on rubrene single crystals with parylene gate insulator." Applied Physics Letters 82(11), 1739-1741.

Podzorov, V., Menard, E., Borissov, A., Kiryukhin, V., Rogers, J. A. and Gershenson, M. E. (2004). "Intrinsic charge transport on the surface of organic semiconductors." Physical Review Letters 93(8), 086602.

Pope, M. and Swenberg, C. E. (1999). Electronic processes in organic crystals and polymers. New York, Oxford University Press.

Samuel, I. D. W. and Turnbull, G. A. (2007). "Organic semiconductor lasers." Chemical Reviews 107, 1272-1295.

Sariciftci, N. S. (1997). Primary Photoexcitations in Conjugated Polymers: Molecular Exciton versus Semiconductor Band Model. Singapore, World Scientific.

Silinsh, E. A. and Capec, V. (1994). Organic molecular crystals: interaction, localization and transport phenomena. New York, American Institute of Physics.

Subramanian, S., Park, S. K., Parkin, S. R., Podzorov, V., Jackson, T. N. and Anthony, J. E. (2008). " Chromophore fluorination enhances crystallization and stability of soluble anthradithiophene semiconductors." Journal of the American Chemical Society 130, 2706-2707.

Thorsmølle, V. K., Averitt, R. D., Chi, X., Hilton, D. J., Smith, D. L., Ramirez, A. P. and Taylor, A. J. (2004). "Ultrafast conductivity dynamics in pentacene probed using terahertz spectroscopy." Applied Physics Letters 84(6), 891-893.

Thorsmølle, V. K., Averitt, R. D., Demsar, J., Smith, D. L., Tretiak, S., Martin, R. L., Chi, X., Crone, B. K., Ramirez, A. P. and Taylor, A. J. (2009). "Morphology effectively controls singlet-triplet exciton relaxation and charge transport in organic semiconductors." Physical Review Letters 102(1), 017401.

Troisi, A. and Orlandi, G. (2006). "Charge-transport regime of crystalline organic semiconductors: diffusion limited by thermal off-diagonal electronic disorder." Physical Review Letters 96, 086601.

Yu, G., Phillips, S. D., Tomozawa, H. and Heeger, A. J. (1990). "Subnanosecond Transient Photoconductivity in Poly(3-Hexylthiophene)." Physical Review B 42(5), 3004-3010. 


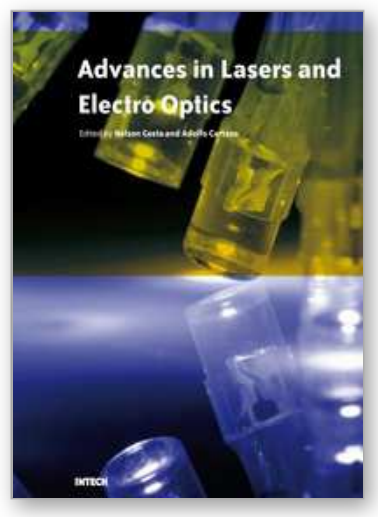

\author{
Advances in Lasers and Electro Optics \\ Edited by Nelson Costa and Adolfo Cartaxo
}

ISBN 978-953-307-088-9

Hard cover, 838 pages

Publisher InTech

Published online 01, April, 2010

Published in print edition April, 2010

Lasers and electro-optics is a field of research leading to constant breakthroughs. Indeed, tremendous advances have occurred in optical components and systems since the invention of laser in the late $50 \mathrm{~s}$, with applications in almost every imaginable field of science including control, astronomy, medicine, communications, measurements, etc. If we focus on lasers, for example, we find applications in quite different areas. We find lasers, for instance, in industry, emitting power level of several tens of kilowatts for welding and cutting; in medical applications, emitting power levels from few milliwatt to tens of Watt for various types of surgeries; and in optical fibre telecommunication systems, emitting power levels of the order of one milliwatt. This book is divided in four sections. The book presents several physical effects and properties of materials used in lasers and electro-optics in the first chapter and, in the three remaining chapters, applications of lasers and electro-optics in three different areas are presented.

\title{
How to reference
}

In order to correctly reference this scholarly work, feel free to copy and paste the following:

Oksana Ostroverkhova, Andrew D. Platt and Whitney E. B. Shepherd (2010). Optical, Photoluminescent, and Photoconductive Properties of Novel High-Performance Organic Semiconductors, Advances in Lasers and Electro Optics, Nelson Costa and Adolfo Cartaxo (Ed.), ISBN: 978-953-307-088-9, InTech, Available from: http://www.intechopen.com/books/advances-in-lasers-and-electro-optics/optical-photoluminescent-andphotoconductive-properties-of-novel-high-performance-organic-semiconduc

\section{INTECH}

open science | open minds

\section{InTech Europe}

University Campus STeP Ri

Slavka Krautzeka 83/A

51000 Rijeka, Croatia

Phone: +385 (51) 770447

Fax: +385 (51) 686166

www.intechopen.com

\section{InTech China}

Unit 405, Office Block, Hotel Equatorial Shanghai

No.65, Yan An Road (West), Shanghai, 200040, China

中国上海市延安西路65号上海国际贵都大饭店办公楼405单元

Phone: +86-21-62489820

Fax: $+86-21-62489821$ 
(C) 2010 The Author(s). Licensee IntechOpen. This chapter is distributed under the terms of the Creative Commons Attribution-NonCommercialShareAlike-3.0 License, which permits use, distribution and reproduction for non-commercial purposes, provided the original is properly cited and derivative works building on this content are distributed under the same license. 